DOI 10.4171/JEMS/188

Filippo Bracci · Manuel D. Contreras · Santiago Díaz-Madrigal

\title{
Pluripotential theory, semigroups and boundary behavior of infinitesimal generators in strongly convex domains
}

Received February 8, 2006 and in revised form June 18, 2007

\begin{abstract}
We characterize infinitesimal generators of semigroups of holomorphic self-maps of strongly convex domains using the pluricomplex Green function and the pluricomplex Poisson kernel. Moreover, we study boundary regular fixed points of semigroups. Among other things, we characterize boundary regular fixed points both in terms of the boundary behavior of infinitesimal generators and in terms of pluripotential theory.
\end{abstract}

Keywords. Semigroups, boundary fixed points, infinitesimal generators, iteration theory, pluripotential theory

\section{Contents}

1. Preliminary results on pluripotential theory in strongly convex domains $\ldots \ldots$. . . . 27

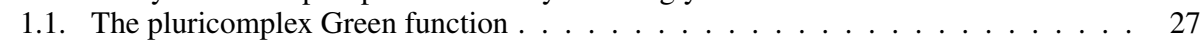

1.2. The pluricomplex Poisson kernel . . . . . . . . . . . . . . . . . . . 28

1.3. Lempert projection devices . . . . . . . . . . . . . . . . . . . . . . . . . . . . 29

2. Iteration theory by means of pluripotential theory $\ldots \ldots \ldots \ldots \ldots$

3. Pluripotential theory and semigroups $\ldots \ldots \ldots \ldots \ldots \ldots$

4. Boundary behavior of infinitesimal generators . . . . . . . . . . . . . . . . . . 41

5. Boundary repelling fixed points and the non-linear resolvent $\ldots \ldots \ldots$. . . . . . . 47

6. Boundary behavior in the unit ball $\ldots \ldots \ldots \ldots \ldots \ldots$. . . . . . . . . . 49

\section{Introduction}

A (continuous) semigroup $\left(\Phi_{t}\right)$ of holomorphic functions on a domain $D \subset \mathbb{C}^{n}$ is a continuous homomorphism from the additive semigroup of non-negative real numbers into the composition semigroup of all holomorphic self-maps of $D$ endowed with the compact-open topology. Namely, the map $[0,+\infty) \ni t \mapsto \Phi_{t} \in \operatorname{Hol}(D, D)$ satisfies the following conditions:

F. Bracci: Dipartimento Di Matematica, Università di Roma "Tor Vergata", Via Della Ricerca Scientifica 1, 00133, Roma, Italy; e-mail: fbracci@mat.uniroma2.it

M. D. Contreras, S. Díaz-Madrigal: Camino de los Descubrimientos, s/n, Departamento de Matemática Aplicada II, Escuela Técnica Superior de Ingenieros, Universidad de Sevilla, 41092 Sevilla, Spain; e-mail: contreras@us.es, madrigal@us.es

Mathematics Subject Classification (2010): Primary 32A99, 32M25; Secondary 31C10 
(1) $\Phi_{0}$ is the identity map id $_{D}$ in $D$,

(2) $\Phi_{t+s}=\Phi_{t} \circ \Phi_{s}$ for all $t, s \geq 0$,

(3) $\Phi_{t}$ tends to id $D$ as $t$ tends to 0 uniformly on compacta of $D$.

It is well known after the basic work of Berkson and Porta [6] in the unit disc $\mathbb{D}=$ $\{\zeta \in \mathbb{C}:|\zeta|<1\}$ that the dependence of every semigroup $\left(\Phi_{t}\right)$ of holomorphic selfmaps of a domain $D \subset \mathbb{C}^{n}$ on the variable $t$ is analytic and to each continuous semigroup $\left(\Phi_{t}\right)$ there corresponds a holomorphic vector field $F: D \rightarrow \mathbb{C}^{n}$ such that $\partial \Phi_{t} / \partial t=$ $F \circ \Phi_{t}$. This vector field $F$ is called the infinitesimal generator of the semigroup $\left(\Phi_{t}\right)$. Conversely, if a holomorphic vector field $F: D \rightarrow \mathbb{C}^{n}$ is semicomplete, that is, for all $z \in D$ its local flow $\gamma^{z}(t)$ such that $\gamma^{z}(0)=z$ is well defined for all $t \geq 0$, then $F$ is the infinitesimal generator of a semigroup of holomorphic self-maps of $D$. We refer to [1] Section 2.5.3] and [32] for more details. Be aware that in the literature there is no standard sign convention for the Cauchy problem generating $F$ : sometimes the problem $\partial \Phi_{t} / \partial t=$ $-F \circ \Phi_{t}$ is considered and thus all formulas regarding $F$ have reverse inequalities with respect to our formulas. For instance, the latter convention is adopted in [3], [5], [19], [21], [22], [31], [32] and [34].

It is clear that the analytical properties of an infinitesimal generator are strictly related to the dynamical and geometrical properties of its semigroup. For instance, any zero of $F$ in $D$ corresponds to a common fixed point for $\left(\Phi_{t}\right)$.

Therefore one of the main questions in the theory of semigroups of holomorphic functions is that of characterizing (in the most useful way) those holomorphic vector fields which are infinitesimal generators. For $D=\mathbb{D}$, the unit disc of $\mathbb{C}$, there is a very nice representation formula, due to Berkson and Porta [6] (see also [1] and [34]). Namely:

Theorem 0.1 (Berkson-Porta). A holomorphic function $G: \mathbb{D} \rightarrow \mathbb{C}$ is the infinitesimal generator of a semigroup $\left(\Phi_{t}\right)$ in $\mathbb{D}$ if and only if there exists a point $b \in \overline{\mathbb{D}}$ and $a$ holomorphic function $p: \mathbb{D} \rightarrow \mathbb{C}$ with $\operatorname{Re} p \geq 0$ such that

$$
G(z)=(z-b)(\bar{b} z-1) p(z), \quad z \in \mathbb{D} .
$$

If the semigroup is not an elliptic group (that is, some/all iterates $\Phi_{t}$ for $t>0$ are elliptic automorphisms), the point $b$ given in Berkson-Porta's formula is exactly the DenjoyWolff point of the semigroup $\left(\Phi_{t}\right)$, that is, $\lim _{t \rightarrow \infty} \Phi_{t}(z)=b$ for all $z \in \mathbb{D}$ (see also Section 2). Other descriptions of infinitesimal generators in $\mathbb{D}$ can be found in [3], [18] and [34, Section 3.6].

In several variables there are various characterizations of infinitesimal generators (see [32] for a good account). All these characterizations reflect the basic fact that holomorphic self-maps of a domain are contractions for the Kobayashi metric of that domain. In fact, Abate [2] proved that if $D$ is a strongly convex domain with smooth boundary and with Kobayashi metric $\kappa_{D}$, then a holomorphic vector field $F: D \rightarrow \mathbb{C}^{n}$ is an infinitesimal generator if and only if $d\left(\kappa_{D} \circ F\right)(z) \cdot F(z) \leq 0$ for all $z \in D$. Unfortunately, even for the case $D=\mathbb{B}^{n}$, the unit ball of $\mathbb{C}^{n}$, such a formula is rather complicated and does not give any information on the dynamical properties of the associated semigroup. Later on, still in this vein, C. de Fabritiis gave a better description of a class of infinitesimal generators 
called "of one-dimensional type" (see [16]). Some rather precise characterizations of infinitesimal generators in the unit balls of infinite-dimensional Hilbert and Banach spaces are given by D. Aharonov, M. Elin, S. Reich, and D. Shoikhet in [3], [4], [22] and [31].

Part of the present paper is devoted to finding characterizations of infinitesimal generators in bounded strongly convex domains with smooth boundary (here and in the rest of the paper "smooth" means at least of class $C^{3}$ ) by means of the pluricomplex Green function $G_{D}$ of Klimek [24], Lempert [28] and Demailly [17] and the pluricomplex Poisson kernel $u_{D, p}$ introduced by Patrizio and the first-named author in [13] (see Section 1 for definitions and preliminaries about pluripotential theory in strongly convex domains). In particular, we prove (see Theorems 3.5 and 3.11):

Theorem 0.2. Let $F: D \rightarrow \mathbb{C}^{n}$ be a holomorphic vector field. The following are equivalent:

(1) The map $F$ is the infinitesimal generator of a semigroup of holomorphic self-maps of $D$.

(2) For all $z, w \in D, z \neq w$, we have $\left.d\left(k_{D}\right)\right|_{(z, w)} \cdot(F(z), F(w)) \leq 0$.

(3) For all $z, w \in D, z \neq w$, we have $\left.d\left(G_{D}\right)\right|_{(z, w)} \cdot(F(z), F(w)) \leq 0$.

(4) For all $z, w \in D$ and for all $r>0$ such that $z-r F(z), w-r F(w) \in D$ we have $k_{D}(z-r F(z), w-r F(w)) \geq k_{D}(z, w)$.

Moreover, if $F$ is $C^{1}$-regular at a point $p \in \partial D$ and $F(z) \neq 0$ for all $z \in D$, then $F$ is an infinitesimal generator whose associated semigroup has Denjoy-Wolff point at $p \in \partial D$ if and only if $d\left(u_{D, p}\right)_{z} \cdot F(z) \leq 0$ for all $z \in D$.

In case $D=\mathbb{B}^{n}$ is the unit ball of $\mathbb{C}^{n}$ (or more generally for the unit ball of complex Hilbert spaces), equivalence between (1) and (4) (and also with an explicit expression of (3), see Remark 3.7) was proven with different methods by Reich and Shoikhet [31, Theorem 2.1]. The last statement can be seen as a Berkson-Porta like formula at the boundary. Moreover, this last formula is just a particular case of a general one for the existence of boundary regular fixed points. We recall that a point $p \in \partial D$ is a boundary regular fixed point-BRFP for short-for a semigroup $\left(\Phi_{t}\right)$ if it is a fixed point for nontangential limits for all $\Phi_{t}$ 's and if the boundary dilatation coefficients at $p$ of the $\Phi_{t}$ 's are all finite (roughly speaking, the boundary dilatation coefficient of a self-map $f$ of $D$ at $p$ is a measure of the velocity with which $f$ approaches $p$ when moving to $p$; see Section 2 for details and precise definitions).

The second part of this paper is devoted to characterizing BRFPs of semigroups in terms of the pluricomplex Poisson kernel $u_{D, p}$ and the local behavior of the infinitesimal generator. In this direction, we cite the following result from [22] (see also [19], [20], [5]):

Theorem 0.3 (Elin-Shoikhet). Let $F: \mathbb{B}^{n} \rightarrow \mathbb{C}^{n}$ be the infinitesimal generator of a semigroup $\left(\Phi_{t}\right)$ in $\mathbb{B}^{n}$ and $p \in \partial \mathbb{B}^{n}$. Assume that $\lim _{(0,1) \ni r \rightarrow 1} F(r p)=0$. The following are equivalent:

(1) $\liminf _{(0,1) \ni r \rightarrow 1} \operatorname{Re}\langle F(r p), p\rangle /(r-1)<+\infty$.

(2) $\lim _{(0,1) \ni r \rightarrow 1}\langle F(r p), p\rangle /(r-1)=\beta$ exists and is finite.

(3) The point $p$ is a BRFP for the semigroup $\left(\Phi_{t}\right)$. 
Moreover, if one of the three conditions holds, then $\beta \in \mathbb{R}$ and the boundary dilatation coefficient of $\Phi_{t}$ at $p$ is $e^{t \beta}$.

The hypothesis in Theorem 0.3 that the infinitesimal generator $F$ has radial limit 0 at $p$, essentially used in the proof of their result, is however not necessary for a point $p$ to be a BRFP (see Example 4.2). Moreover, surprisingly enough, Theorem 0.3 would be false without such a hypothesis (see Example 4.3 where we construct an infinitesimal generator for which (1) holds at some $p \in \partial \mathbb{B}^{2}$ but $p$ is not a BRFP for the associated semigroup). In fact, it turns out that a point $p \in \partial \mathbb{B}^{n}$ is a BRFP for the semigroup if and only if a condition similar to (1) holds not just for the radial direction but for all the directions. To be more precise and in order to state the result for general strongly convex domains, we need to use the so called Lempert projection devices. For the time being, we can say that a Lempert projection device $\left(\varphi, \widetilde{\rho}_{\varphi}\right)$ is given by a particular holomorphic map $\varphi: \mathbb{D} \rightarrow D$ (called complex geodesic) which extends smoothly to $\partial \mathbb{D}$ and a holomorphic map $\tilde{\rho}_{\varphi}: D \rightarrow \mathbb{D}$ such that $\tilde{\rho}_{\varphi} \circ \varphi=\mathrm{id}_{\mathbb{D}}$ (actually a Lempert projection device is a triple of maps; we refer the reader to Section 1 for details). For the unit ball $\mathbb{B}^{n}$ a Lempert projection device $\left(\varphi, \widetilde{\rho}_{\varphi}\right)$ is nothing but a (suitable) parametrization $\varphi: \mathbb{D} \rightarrow \mathbb{B}^{n}$ of the intersection of $\mathbb{B}^{n}$ with an affine complex line and $\tilde{\rho}_{\varphi}$ is the orthogonal projection on it (see also Section 6 where the case of $\mathbb{B}^{n}$ is studied in detail). Our second main result is the following:

Theorem 0.4. Let $D \subset \mathbb{C}^{n}$ be a bounded strongly convex domain with smooth boundary, let $F$ be the infinitesimal generator of a semigroup $\left(\Phi_{t}\right)$ of holomorphic self-maps of $D$, and $p \in \partial D$. The following are equivalent:

(1) The semigroup $\left(\Phi_{t}\right)$ has a BRFP at $p$ with boundary dilatation coefficients $\alpha_{t}(p) \leq$ $e^{\beta t}$ for all $t \geq 0$.

(2) There exists $\bar{\beta} \in \mathbb{R}$ such that $d\left(u_{D, p}\right)_{z} \cdot F(z)+\beta u_{D, p}(z) \leq 0$ for all $z \in D$.

(3) There exists $C>0$ such that for any Lempert projection device $\left(\varphi, \tilde{\rho}_{v}\right)$ with $\varphi(1)=p$,

$$
\limsup _{(0,1) \ni r \rightarrow 1} \frac{\left|d\left(\tilde{\rho}_{\varphi}\right)_{\varphi(r)} \cdot F(\varphi(r))\right|}{1-r} \leq C
$$

Moreover, if $p$ is a BRFP for $\left(\Phi_{t}\right)$ with boundary dilatation coefficients $\alpha_{t}(p)=e^{-b t}$ then

$$
b=\inf _{z \in D} \frac{d\left(u_{D, p}\right)_{z} \cdot F(z)}{u_{d, p}(z)}
$$

and the non-tangential limit

$$
A(\varphi, p):=\angle \lim _{\zeta \rightarrow 1} \frac{d\left(\widetilde{\rho}_{\varphi}\right)_{\varphi(\zeta)} \cdot F(\varphi(\zeta))}{\zeta-1}
$$

exists, $A(\varphi, p) \in \mathbb{R}$ and $A(\varphi, p) \leq b$. Also, $b=\sup A(\varphi, p)$, with the supremum taken as $\varphi$ varies among all Lempert projection devices $\left(\varphi, \widetilde{\rho}_{v}\right)$ with $\varphi(1)=p$. 
This result is contained in Theorems 3.8 and 4.7. One of the main ingredients in the proof is the remarkable property that the projection of an infinitesimal generator on every complex geodesic is still an infinitesimal generator.

In order to give the proof of the previous results, in the first section we recall pluripotential theory in strongly convex domains and in the second section we study iteration using the pluricomplex Green function and the pluricomplex Poisson kernel. We should say that even if part of the results in Section 2 are already known, our present formulation seems to be new and, as we will prove later, quite effective. Section 3 is devoted to the interactions between pluripotential theory and semigroups. In Section 4 we discuss a couple of examples on the boundary behavior of semigroups and complete the proof of our characterization of BRFPs in terms of the boundary behavior of the infinitesimal generator. As a consequence, in Corollary 4.8 we discuss stationary points of semigroups (i.e. those BRFPs for which the boundary dilatation coefficient is less than or equal to 1). In Section 5 we consider the non-linear resolvent of Reich and Shoikhet, proving that every BRFP of the non-linear resolvent is a BRFP for the semigroup (see Proposition 5.2). Finally, in Section 6 we translate our results to the ball $\mathbb{B}^{n}$ where some more explicit formulations, using automorphisms, are possible. In this case, we also discuss the boundary behavior of the infinitesimal generator at a BRFP under some boundedness conditions (see Corollary 6.2).

\section{Preliminary results on pluripotential theory in strongly convex domains}

For the definition, properties and further results about strongly convex domains, we refer the reader to the nice monograph by Abate [1, Part 2]. Likewise, for an introduction to pluripotential theory with a special emphasis on complex Monge-Ampère operators, we recommend the beautiful book by Klimek [25] (a short introduction is also contained in [10]). Anyhow, for the sake of clarity, we are going to give some basic definitions and define the tools we need later on.

\subsection{The pluricomplex Green function}

Let $D \subset \subset \mathbb{C}^{n}$ be a domain and $z \in D$. Define

$$
\mathcal{K}_{D, z}=\{u \text { plurisubharmonic in } D: u<0, u(w)-\log \|z-w\| \leq O(1) \text { as } w \rightarrow z\} .
$$

Klimek's pluricomplex Green function [24] is defined as

$$
G_{D}(z, w):=\sup _{u \in \mathcal{K}_{D, z}} u(w) .
$$

The function $w \mapsto G_{D}(z, w)$ is plurisubharmonic in $D$, locally bounded in $D \backslash\{z\}$ and has a logarithmic pole at $z$ (see [24] and [25]). If $D$ is hyperconvex (in particular, if $D$ is a convex domain), then Demailly [17] showed that $G_{D}$, extended to be 0 on $D \times \partial D$, is continuous as a function $G_{D}: D \times \bar{D} \rightarrow[-\infty, 0)$. Moreover, from the work of Lempert 
[28] and Demailly [17], it turns out that $G_{D}(z, \cdot)$ is the unique solution of the following homogeneous Monge-Ampère equation:

$$
\begin{cases}u \text { plurisubharmonic in } D, & \\ (\partial \bar{\partial} u)^{n}=0 & \text { in } D \backslash\{z\}, \\ \lim _{w \rightarrow x} u(w)=0 & \text { for all } x \in \partial D, \\ u(w)-\log |w-z|=O(1) & \text { as } w \rightarrow z .\end{cases}
$$

By the very definition, if $h: D \rightarrow D^{\prime}$ is holomorphic, then for all $z, w \in D$,

$$
G_{D^{\prime}}(h(z), h(w)) \leq G_{D}(z, w) .
$$

In case $D$ is a bounded strongly convex domain with smooth boundary (here and in the rest of the paper "smooth" means at least of class $C^{3}$ ) Lempert [28] proved that $G_{D}(z, w)$ is smooth and regular for $(z, w) \in \bar{D} \times \bar{D} \backslash \operatorname{Diag}(\bar{D} \times \bar{D})$ and that

$$
G_{D}(z, w)=\log \tanh k_{D}(z, w),
$$

where $k_{D}(z, w)$ is the Kobayashi distance of $D$ (for definition and properties we refer to [1] or [26]).

For instance, for $D=\mathbb{D}$, the unit disc in $\mathbb{C}$, the pluricomplex Green function coincides with the usual (negative) Green function, while for $D=\mathbb{B}^{n}$, the unit ball of $\mathbb{C}^{n}$, we have

$$
G_{\mathbb{B}^{n}}(z, w)=\log \left\|T_{z}(w)\right\|,
$$

where $T_{z}: \mathbb{B}^{n} \rightarrow \mathbb{B}^{n}$ is any automorphism of $\mathbb{B}^{n}$ with the property that $T_{z}(z)=0$.

\subsection{The pluricomplex Poisson kernel}

Let $D \subset \subset \mathbb{C}^{n}$ be a strongly convex domain with smooth boundary, $z_{0} \in D$ and let $p \in \partial D$. In [13], Patrizio and the first author introduced a plurisubharmonic function $u_{D, p}: D \rightarrow(-\infty, 0)$ which extends smoothly on $\bar{D} \backslash\{p\}$ such that $d\left(u_{D, p}\right)_{z} \neq 0$ for all $z \in D, u_{D, p}(q)=0$ for all $q \in \partial D \backslash\{p\}$ and $u_{D, p}$ has a simple pole at $p$ along nontangential directions. Up to a real positive multiple, we assume here that $u_{D, p}\left(z_{0}\right)=-1$. The function $u_{D, p}$ solves the following homogeneous Monge-Ampère equation:

$$
\begin{cases}u \text { plurisubharmonic } & \text { in } D, \\ (\partial \bar{\partial} u)^{n}=0 & \text { in } D, \\ u<0 & \text { in } D, \\ u(w)=0 & \text { for all } w \in \partial D \backslash\{p\}, \\ u(w) \approx\|w-p\|^{-1} & \text { as } w \rightarrow p \text { non-tangentially. }\end{cases}
$$

In [13] and [14], the authors prove that $u_{D, p}$ shares many properties with the classical Poisson kernel for the unit disc. In case $D=\mathbb{D}$, the function $u_{\mathbb{D}, p}$ (normalized so that 
$\left.u_{\mathbb{D}, p}(0)=-1\right)$ is in fact the classical (negative) Poisson kernel. In case $D=\mathbb{B}^{n}$, the pluricomplex Poisson kernel (normalized so that $u_{\mathbb{B}^{n}, p}(0)=-1$ ) is given by

$$
u_{\mathbb{B}^{n}, p}(z)=-\frac{1-\|z\|^{2}}{|\langle p-z, p\rangle|^{2}} .
$$

The level sets of $u_{D, p}$ are exactly boundaries of Abate's horospheres. Recall that a horosphere $E_{D}(p, R)$ of center $p \in \partial D$ and radius $R>0$ (with respect to $z_{0}$ ) is given by

$$
E_{D}(p, R)=\left\{z \in D: \lim _{w \rightarrow p}\left[k_{D}(z, w)-k_{D}\left(z_{0}, w\right)\right]<\frac{1}{2} \log R\right\} .
$$

Notice that the existence of the limit in the definition of $E_{D}(p, R)$ is a characteristic of smooth strongly convex domains and follows again from Lempert's theory (see [1, Theorem 2.6.47]). Thanks to our normalization $u_{D, p}\left(z_{0}\right)=-1$, it follows that

$$
E_{D}(p, R)=\left\{z \in D: u_{D, p}(z)<-1 / R\right\} .
$$

For the unit disc, these level sets are boundaries of horocycles and, in case $D=\mathbb{B}^{n}$, these are boundaries of horospheres in $\mathbb{B}^{n}$ with center $p$, whose explicit expression is

$$
E_{\mathbb{B}^{n}}(p, R)=\left\{z \in \mathbb{B}^{n}: \frac{|1-\langle z, p\rangle|^{2}}{1-\|z\|^{2}}<R\right\} .
$$

More information about the properties of $u_{D, p}$ (such as smooth dependence on $p$, extremality, uniqueness, relations with the pluricomplex Green function, usage in representation formulas for pluriharmonic functions) can be found in [14].

\subsection{Lempert projection devices}

We recall that a complex geodesic $\varphi: \mathbb{D} \rightarrow D$ is a holomorphic isometry between $k_{\mathbb{D}}$ (the hyperbolic distance in $\mathbb{D}$ ) and $k_{D}$. By Lempert's work (see [28] and [1]) given two points $z_{0} \in D$ and $z \in \bar{D}$, there exists a unique complex geodesic $\varphi: \mathbb{D} \rightarrow D$ such that $\varphi$ extends smoothly past the boundary, $\varphi(0)=z_{0}$ and $\varphi(t)=z$, with $t \in(0,1)$ if $z \in D$ and $t=1$ if $z \in \partial D$. Moreover, for any such complex geodesic there exists a holomorphic retraction $\rho_{\varphi}: D \rightarrow \varphi(\mathbb{D})$, i.e. there exists a holomorphic map $\rho_{\varphi}: D \rightarrow D$ such that $\rho_{\varphi} \circ \rho_{\varphi}=\rho_{\varphi}$ and $\rho_{\varphi}(z)=z$ for any $z \in \varphi(\mathbb{D})$.

Given a complex geodesic, there might exist many holomorphic retractions to such geodesic, but the one constructed by Lempert turns out to be the only one with affine fibers (see [14, Section 3]). We call such a $\rho_{\varphi}$ the Lempert projection associated with $\varphi$.

Furthermore, we let $\widetilde{\rho}_{\varphi}:=\varphi^{-1} \circ \rho_{\varphi}: D \rightarrow \mathbb{D}$ and call it the left inverse of $\varphi$, for $\tilde{\rho}_{\varphi} \circ \varphi=\mathrm{id} \mathbb{D}$. The triple $\left(\varphi, \rho_{\varphi}, \tilde{\rho}_{\varphi}\right)$ is called a Lempert projection device.

For $D=\mathbb{B}^{n}$ the image of the complex geodesic through the points $z \neq w \in \overline{\mathbb{B}^{n}}$ is just the one-dimensional slice $S_{z, w}:=\mathbb{B}^{n} \cap\{z+\zeta(z-w): \zeta \in \mathbb{C}\}$. The Lempert projection is thus given by the orthogonal projection of $\mathbb{B}^{n}$ onto $S_{z, w}$. 
By Lempert's very definition, if $\varphi: \mathbb{D} \rightarrow D$ is a complex geodesic, then

$$
G_{D}(\varphi(\zeta), \varphi(\eta))=G_{\mathbb{D}}(\zeta, \eta) \quad \text { for all } \zeta, \eta \in \mathbb{D}
$$

Finally, we mention [13, p. 516] that for any given Lempert projection device $\left(\varphi, \rho_{\varphi}, \widetilde{\rho}_{\varphi}\right)$ in $D$ with $\varphi(1)=p$ there exists $a_{\varphi}>0$ such that for all $\zeta \in \mathbb{D}$,

$$
u_{D, p}(\varphi(\zeta))=a_{\varphi} u_{\mathbb{D}, 1}(\zeta)
$$

\section{Iteration theory by means of pluripotential theory}

Both the pluricomplex Green function and the pluricomplex Poisson kernel can be used to describe dynamical properties of holomorphic self-maps of a bounded strongly convex domain with smooth boundary. The aim of this section is to formulate precisely the results we need later on in terms of pluripotential theory.

All the results presented in this section are strongly based on some known results about iteration (mainly due to Abate, see [1]). However, for completeness, we sometimes provide a sketch of some new direct proofs.

For a map $h: D \rightarrow D$ we denote by $\operatorname{Fix}(h)$ the set of its fixed points in $D$,

$$
\operatorname{Fix}(h):=\{z \in D: h(z)=z\} .
$$

To begin with, we can reformulate a Schwarz-type lemma for strongly convex domains as follows:

Theorem 2.1. Let $D \subset \subset \mathbb{C}^{n}$ be a strongly convex domain with smooth boundary and let $z_{0} \in D$. Let $h: D \rightarrow D$ be holomorphic. Then $h\left(z_{0}\right)=z_{0}$ if and only if for all $z \in D$,

$$
G_{D}\left(z_{0}, h(z)\right) \leq G_{D}\left(z_{0}, z\right) .
$$

Moreover, if equality holds in 2.1) for some $z \neq z_{0}$ and $\varphi: \mathbb{D} \rightarrow D$ is the complex geodesic such that $\varphi(0)=z_{0}$ and $\varphi(t)=z$ for some $t \in(0,1)$, then $h \circ \varphi: \mathbb{D} \rightarrow D$ is a complex geodesic and $h: \varphi(\mathbb{D}) \rightarrow h(\varphi(\mathbb{D}))$ is an automorphism.

Proof. The necessity and sufficiency of 2.1) follow directly from the very definition of $G_{D}$ and 1.1 .

In order to prove the last statement, assume that $G_{D}\left(z_{0}, h(z)\right)=G_{D}\left(z_{0}, z\right)$ for some $z \in D, z \neq z_{0}$. Let $\left(\varphi, \rho_{\varphi}, \tilde{\rho}_{\varphi}\right)$ be the Lempert projective device such that $\varphi(0)=z_{0}$ and $\varphi(t)=z$ for some $t \in(0,1)$ and let $\left(\psi, \rho_{\psi}, \tilde{\rho}_{\psi}\right)$ be the Lempert projective device such that $\psi(0)=z_{0}$ and $\psi(r)=h(z)$ for some $r \in(0,1)$. Let $\tilde{h}(\zeta):=\tilde{\rho}_{\psi}(h(\varphi(\zeta)))$ for $\zeta \in \mathbb{D}$. Notice that $\tilde{h}(0)=0$. Then $H(\zeta):=G_{\mathbb{D}}(0, \tilde{h}(\zeta))-G_{\mathbb{D}}(0, \zeta) \leq 0$. Moreover, the function $\mathbb{D} \ni \zeta \mapsto H(\zeta)$ is harmonic on $\mathbb{D} \backslash\left\{0, \tilde{h}^{-1}(0)\right\}$ and bounded from above, thus can be extended in a subharmonic way to all of $\mathbb{D}$. We continue to denote such an extension by $H$. For all $\zeta \in \mathbb{D}$, by $(1.5)$, it follows that

$$
G_{\mathbb{D}}(0, \tilde{h}(t))=G_{D}(\psi(0), \psi(\tilde{h}(t)))=G_{D}\left(z_{0}, h(z)\right)=G_{D}\left(z_{0}, z\right)=G_{\mathbb{D}}(0, t) .
$$

By the maximum principle then $H(\zeta) \equiv 0$ and $\tilde{h}(\zeta)=e^{i \theta} \zeta$ for some $\theta \in \mathbb{R}$, proving the statement. 
Definition 2.2. Let $D \subset \subset \mathbb{C}^{n}$ be a strongly convex domain with smooth boundary, $p \in$ $\partial D$ and $h: D \rightarrow D$ holomorphic. The boundary dilatation coefficient $\alpha_{h}(p) \in(0,+\infty]$ is defined as

$$
\alpha_{h}(p)=\inf _{q \in \partial D}\left\{\sup _{z \in D} \frac{u_{D, p}(z)}{u_{D, q}(h(z))}\right\} .
$$

As we show, this number can be characterized in several ways. Some of them are widely used in the literature (see [1] and [9]). Indeed, we have:

Proposition 2.3. Let $D \subset \subset \mathbb{C}^{n}$ be a strongly convex domain with smooth boundary, $p \in \partial D$ and $h: D \rightarrow D$ holomorphic. Then the following are equivalent:

(1) $\alpha_{h}(p)<+\infty$.

(2) There exist a (necessarily unique) point $q \in \partial D$ and a number $\lambda>0$ such that

$$
h\left(E_{D}(p, R)\right) \subseteq E_{D}(q, \lambda R) \quad \text { for all } R>0 .
$$

(3) We have

$$
\frac{1}{2} \log \beta_{h}(p):=\liminf _{z \rightarrow p}\left[k_{D}\left(z, z_{0}\right)-k_{D}\left(h(z), z_{0}\right)\right]<+\infty .
$$

Moreover, if one of the statements holds, then

$$
\left.\beta_{h}(p)=\alpha_{h}(p)=\inf \{\lambda>0: \lambda \text { satisfies } 2.2\}\right\} .
$$

Proof. By the very definition, (1) is equivalent to the existence of $q \in \partial D$ such that $u_{D, q}(h(z)) \leq\left(1 / \alpha_{h}(p)\right) u_{D, p}(z)$ for all $z \in D$. By $(1.4),(1)$ and (2) are equivalent and $\alpha_{h}(p)=\inf \{\lambda>0: \lambda$ satisfies $[2.2)\}$.

If (3) holds then (2) follows from Abate's version of the Julia lemma for strongly convex domains (see [1, Theorem 2.4.16]); also by the same token, $\beta_{h}(p) \geq \inf \{\lambda>0$ : $\lambda$ satisfies 2.2 \}.

Finally, if (2) holds, let $\varphi: \mathbb{D} \rightarrow D$ be the complex geodesic such that $\varphi(0)=z_{0}$ and $\varphi(1)=p$ and let $\tilde{\rho}_{\varphi}: D \rightarrow \mathbb{D}$ be its left inverse. Let $\tilde{h}:=\tilde{\rho}_{\varphi} \circ h \circ \varphi: \mathbb{D} \rightarrow \mathbb{D}$. Since $\varphi$ is an isometry between the Poincaré distance of $\mathbb{D}$ and the Kobayashi distance of $D$ and $k_{\mathbb{D}}\left(\widetilde{\rho}_{\varphi}(z), \tilde{\rho}_{\varphi}(w)\right) \leq k_{D}(z, w)$ for all $z, w \in D$, it is easy to check that for all $R>0$ we have $\tilde{h}\left(E_{\mathbb{D}}(1, R)\right) \subseteq E_{\mathbb{D}}(1, \lambda R)$. Therefore the classical Julia-Wolff-Carathéodory theorem implies that $\beta_{\tilde{h}}(1)<\infty$ and actually $\beta_{\tilde{h}}(1) \leq \lambda$. Now,

$$
\begin{aligned}
\frac{1}{2} \log \beta_{h}(p) & =\liminf _{w \rightarrow p}\left[k_{D}(w, \varphi(0))-k_{D}(h(w), \varphi(0))\right] \\
& \leq \liminf _{\zeta \rightarrow 1}\left[k_{D}(\varphi(\zeta), \varphi(0))-k_{D}(h(\varphi(\zeta)), \varphi(0))\right] \\
& \leq \liminf _{\zeta \rightarrow 1}\left[k_{\mathbb{D}}(\zeta, 0)-k_{\mathbb{D}}\left(\widetilde{\rho}_{\varphi}(h(\varphi(\zeta))), 0\right)\right]=\frac{1}{2} \log \beta_{\tilde{h}}(1)
\end{aligned}
$$

which proves that $\beta_{h}(p)<+\infty$ and actually $\beta_{h}(p) \leq \inf \{\lambda>0: \lambda$ satisfies 2.2$\}$, ending the proof of the proposition. 
It is worth mentioning that by our very definition $\alpha_{h}(p)$ does not depend on $z_{0}$, while a priori the liminf in 2.3 does. However, the independence of this liminf from $z_{0}$ can also be shown directly (see [9, Lemma 6.1]).

We have the following version of Julia's lemma for strongly convex domains:

Theorem 2.4. Let $D \subset \subset \mathbb{C}^{n}$ be a strongly convex domain with smooth boundary, $p \in$ $\partial D$ and $h: D \rightarrow D$ holomorphic. If $\alpha_{h}(p)<+\infty$, then there exists a unique point $q \in \partial D$ such that $h$ has non-tangential limit $q$ at $p$ and, for all $z \in D$,

$$
u_{D, q}(h(z)) \leq \frac{1}{\alpha_{h}(p)} u_{D, p}(z)
$$

Moreover, if equality holds in 2.6 for some $z \in D$ and $\varphi: \mathbb{D} \rightarrow D$ is the complex geodesic such that $\varphi(1)=p$ and $\varphi(0)=z$, then $h \circ \varphi: \mathbb{D} \rightarrow D$ is a complex geodesic and $h: \varphi(\mathbb{D}) \rightarrow h(\varphi(\mathbb{D}))$ is an automorphism.

Proof. By the very definition, if $\alpha_{h}(p)<+\infty$ then there exists at least one $q \in \partial D$ and a constant $C>0$ such that

$$
u_{D, q}(h(z)) \leq C u_{D, p}(z) \quad \text { for all } z \in D .
$$

Since $u_{D, p}$ has a simple pole as $z \rightarrow p$ along non-tangential directions, the above inequality (2.7) implies that $h$ has non-tangential limit $q$ at $p$. In particular, this implies that there exists at most one $q \in \partial D$ such that $\sup _{z \in D} u_{D, p}(z) / u_{D, q}(h(z))<+\infty$. Therefore, (2.6) holds.

In order to prove the last statement, assume that $u_{D, q}(h(z))=\left(1 / \alpha_{h}(p)\right) u_{D, p}(z)$ for some $z \in D$. Let $\left(\varphi, \rho_{\varphi}, \widetilde{\rho}_{\varphi}\right)$ be the Lempert projective device such that $\varphi(1)=p$ and $\varphi(0)=z$ and let $\left(\psi, \rho_{\psi}, \widetilde{\rho}_{\psi}\right)$ be the Lempert projective device such that $\psi(1)=q$ and $\psi(0)=h(z)$. Write $\tilde{h}(\zeta):=\tilde{\rho}_{\psi}(h(\varphi(\zeta)))$ for $\zeta \in \mathbb{D}$. By 1.6 and 2.6 it follows that $H(\zeta):=u_{\mathbb{D}, 1}(\zeta)-\lambda u_{\mathbb{D}, 1}(\tilde{h}(\zeta)) \leq 0$ for $\zeta \in \mathbb{D}$ and $\lambda:=\alpha_{h}(p) a_{\psi} / a_{\varphi}$. The function $H$ is harmonic in $\mathbb{D}$ and, by construction,

$$
H(0)=u_{\mathbb{D}, 1}(0)-\lambda u_{\mathbb{D}, 1}(\tilde{h}(0))=\frac{1}{a_{\varphi}} u_{D, p}(z)-\frac{\lambda}{a_{\psi}} u_{D, q}(h(z))=0 .
$$

Thus the maximum principle implies that $H(\zeta) \equiv 0$, which in turns implies that $\lambda=1$ and $\tilde{h}$ is the identity on $\mathbb{D}$, and the statement follows.

Let $D \subset \mathbb{C}^{n}$ be a bounded strongly convex domain with smooth boundary, let $z_{0} \in D$ and let $p \in \partial D$. Following Abate [1] we denote by $K(p, R)$ the $K$-region with vertex $p$ and radius $R>1$ defined as

$$
K(p, R)=\left\{z \in D: \lim _{w \rightarrow p}\left[k_{D}(z, w)-k_{D}\left(z_{0}, w\right)\right]+k_{D}\left(z, z_{0}\right)<\log R\right\} .
$$

If $Q: D \rightarrow \mathbb{C}^{n}$ is a function, we write $\mathrm{K}-\lim _{z \rightarrow p} Q(z)=L$ if for any sequence $\left\{z_{k}\right\} \subset D$ which tends to $p$ and belongs eventually to a K-region $K(p, R)$ for some $R>1$, we have $\lim _{k \rightarrow \infty} Q\left(z_{k}\right)=L$. Notice that if $Q$ has K-limit $L$ at $p$ then in particular it has non-tangential limit $L$ at $p$. 
Remark 2.5. If $\alpha_{h}(p)<+\infty$ and $q \in \partial D$ is the point given by Theorem 2.4 then actually $h$ has K-limit $q$ at $p$. This follows from Abate's version of the classical JuliaWolff-Carathéodory theorem, but also from (2.6), since actually $u_{D, p}(z) \rightarrow-\infty$ when $z \rightarrow p$ inside a K-region (see [14, Section 5]).

The reason for the importance of boundary dilatation coefficients in iteration theory is that while they give a global picture of the dynamics of a self-map of $D$, they can easily be computed as radial limits along any complex geodesic. We are going to state this fact in a particular case which we need later. Before that we give the following

Definition 2.6. Let $D \subset \mathbb{C}^{n}$ be a strongly convex domain with smooth boundary. Let $h: D \rightarrow D$ be holomorphic. We say that a point $p \in \partial D$ is a boundary regular fixed point, BRFP for short, if $h$ has non-tangential limit $p$ at $p$ and the boundary dilatation coefficient $\alpha_{h}(p)$ is finite. A BRFP with boundary dilatation coefficient $\leq 1$ is also called $a$ stationary point. Likewise, those boundary regular fixed points with $\alpha_{h}(p)>1$ are usually called boundary repelling fixed points.

Now we can state the following version of the Julia-Wolff-Carathéodory theorem, due essentially to Abate:

Theorem 2.7. Let $D \subset \subset \mathbb{C}^{n}$ be a strongly convex domain with smooth boundary. Let $h: D \rightarrow D$ be holomorphic and let $p \in \partial D$. Then $p$ is a BRFP for $h$ if and only if for some-and hence any-Lempert projective device $\left(\varphi, \rho_{\varphi}, \tilde{\rho}_{\varphi}\right)$ such that $\varphi(1)=p$,

$$
\liminf _{(0,1) \ni r \rightarrow 1} \frac{\left|1-\tilde{\rho}_{\varphi}(h(\varphi(r)))\right|}{1-r}<+\infty .
$$

Moreover, if $p$ is a BRFP for $h$ then

$$
\lim _{r \rightarrow 1} \frac{1-\widetilde{\rho}_{\varphi}(h(\gamma(r)))}{1-\widetilde{\rho}_{\varphi}(\gamma(r))}=\alpha_{h}(p)
$$

for any curve $\gamma:[0,1) \rightarrow D$ such that $\lim _{r \rightarrow 1} \gamma(r)=p$, the curve in $\mathbb{D}$ given by $r \mapsto \tilde{\rho}_{\varphi}(\gamma(r))$ converges non-tangentially to 1 and $\lim _{r \rightarrow 1} k_{D}\left(\gamma(r), \rho_{\varphi}(\gamma(r))\right)=0$. In particular, the map $\widetilde{\rho}_{\varphi} \circ h \circ \varphi: \mathbb{D} \rightarrow \mathbb{D}$ has BRFP at 1 with boundary dilatation coefficient $\alpha_{h}(p)$.

Proof. If $p$ is a BRFP for $h$ then the result follows from [1, Theorem 2.7.14].

Conversely, assume (2.8) holds. Then

$$
\liminf _{\zeta \rightarrow 1} \frac{1-\left|\widetilde{\rho}_{\varphi}(h(\varphi(\zeta)))\right|}{1-|\zeta|}<\liminf _{(0,1) \ni r \rightarrow 1} \frac{\left|1-\widetilde{\rho}_{\varphi}(h(\varphi(r)))\right|}{1-r}<+\infty .
$$

Thus the classical Julia-Wolff-Carathéodory theorem (see, e.g., [1]) implies that 1 is a BRFP for $\zeta \mapsto \widetilde{\rho}_{\varphi}(h(\varphi(\zeta)))$ with boundary dilatation coefficient $a<+\infty$. Now, by 2.3), taking into account that $k_{\mathbb{D}}\left(\widetilde{\rho}_{\varphi}(z), \widetilde{\rho}_{\varphi}(w)\right) \leq k_{D}(z, w)$ and arguing as in 2.5) we find that $\frac{1}{2} \log \alpha_{h}(p) \leq \frac{1}{2} \log a$, so that $\alpha_{h}(p)<+\infty$. Theorem 2.4 implies that $h$ has non-tangential limit $q$ at $p$ for some $q \in \partial D$. In order to end the proof we need to show 
that $q=p$. To this end, we first notice that $\lim _{r \rightarrow 1} \tilde{\rho}_{\varphi}(h(\varphi(r)))=1$ forces $h(\varphi(r))$ to tend to $p$ as $r \rightarrow 1$ because $\tilde{\rho}_{\varphi}(\bar{D} \backslash\{\varphi(\overline{\mathbb{D}})\}) \subset \mathbb{D}$ by [29. Proposition 1, p. 345]. But $\varphi(\mathbb{D})$ is transverse to $\partial D$ by Hopf's lemma and therefore $\varphi(r) \rightarrow p$ non-tangentially. This implies that $\angle \lim _{z \rightarrow p} h(z)=p$, and we are done.

In case a holomorphic self-map of $D$ has no fixed points in $D$, there always exists a particular stationary point (see [1, Theorem 2.4.23]):

Theorem 2.8 (Abate). Let $D \subset \subset \mathbb{C}^{n}$ be a strongly convex domain with smooth boundary. Let $h: D \rightarrow D$ be holomorphic. If $\operatorname{Fix}(h)=\emptyset$ then there exists a unique point $p \in \partial D$, called the Denjoy-Wolff point of $h$, such that $p$ is a stationary point for $h$ and the sequence of iterates $\left\{h^{\circ m}\right\}$ converges uniformly on compacta to the constant map $D \ni z \mapsto p$.

Stationary points are quite special, as the following proposition shows:

Proposition 2.9. Let $D \subset \subset \mathbb{C}^{n}$ be a strongly convex domain with smooth boundary. Let $h: D \rightarrow D$ be holomorphic. Assume that $p \in \partial D$ is a stationary point.

(1) If $\operatorname{Fix}(h) \neq \varnothing$ then there exists a complex geodesic $\varphi: \mathbb{D} \rightarrow D$ such that $\varphi(1)=p$ and $\varphi(D) \subseteq \operatorname{Fix}(h)$. Moreover, for all $\theta \in \mathbb{R}$, the point $\varphi\left(e^{i \theta}\right) \in \partial D$ is a stationary point for $h$ and $\alpha_{h}\left(\varphi\left(e^{i \theta}\right)\right)=1$.

(2) If $\operatorname{Fix}(h)=\emptyset$ then $p$ is the Denjoy-Wolff point of $h$ and $h$ has no other stationary point in $\partial D$.

Proof. (1) Assume $z \in \operatorname{Fix}(h)$. Let $\varphi: \mathbb{D} \rightarrow D$ be the complex geodesic such that $\varphi(0)=$ $z$ and $\varphi(1)=p$. Consider the holomorphic self-map of the unit disc $\psi(\zeta):=\widetilde{\rho}_{\varphi} \circ h \circ \varphi(\zeta)$. Then $\psi(0)=0$ and by Theorem 2.7, $\psi$ has a stationary point at 1 . But then by the Herzig theorem [23] (see also the classical Wolff Lemma in [1]) it follows that $\psi(\zeta) \equiv \zeta$. Thus for any $\zeta, \xi \in \mathbb{D}$,

$$
\begin{aligned}
k_{D}(\varphi(\zeta), \varphi(\xi)) & \geq k_{D}(h(\varphi(\zeta)), h(\varphi(\xi))) \geq k_{D}\left(\rho_{\varphi}(h(\varphi(\zeta))), \rho_{\varphi}(h(\varphi(\xi)))\right) \\
& =k_{D}(\varphi(\psi(\zeta)), \varphi(\psi(\xi)))=k_{D}(\varphi(\zeta), \varphi(\xi))=k_{\mathbb{D}}(\zeta, \xi)
\end{aligned}
$$

forcing equality at all the steps. In particular, $h \circ \varphi: \mathbb{D} \rightarrow D$ is a complex geodesic such that $h(\varphi(0))=z$ and $h(\varphi(1))=p$. By the uniqueness of complex geodesics passing through two given points of $\bar{D}$ it follows that $h \circ \varphi=\varphi$. Hence $\varphi(\mathbb{D}) \subset \operatorname{Fix}(h)$.

Assertion (2) follows similarly. Indeed, let $q \in \partial D$ be the Denjoy-Wolff point of $h$. If $q \neq p$ then consider the complex geodesic $\varphi: \mathbb{D} \rightarrow D$ such that $\varphi(-1)=q$ and $\varphi(1)=p$ and let $\psi(\zeta):=\tilde{\rho}_{\varphi} \circ h \circ \varphi(\zeta)$. As before Theorem 2.7 implies that $\psi$ has stationary points at -1 and +1 . Now the Wolff Lemma implies that $\psi(\zeta) \equiv \zeta$. Then we can proceed exactly as before to obtain $h(\varphi(\zeta))=\varphi(\zeta)$ for all $\zeta \in \mathbb{D}$, contradicting the hypothesis. 


\section{Pluripotential theory and semigroups}

The aim of this section is to use the pluricomplex Green function and the pluricomplex Poisson kernel to characterize infinitesimal generators of semigroups of holomorphic selfmaps of a strongly convex domain and their dynamical properties.

We start by recalling the following result (see [5] for $D=\mathbb{B}^{n}$ and [1, Theorem 2.5.24], [11, Theorem A.1] for the general case).

Theorem 3.1. Let $D \subset \mathbb{C}^{n}$ be a bounded strongly convex domain with smooth boundary. Let $\left(\Phi_{t}\right)$ be a one-parameter semigroup of holomorphic self-maps of $D$. Then either

- $\bigcap_{t \geq 0} \operatorname{Fix}\left(\Phi_{t}\right) \neq \emptyset$, or

- Fix $\left(\Phi_{t}\right)=\emptyset$ for all $t>0$, there exists a unique $\tau \in \partial D$ such that $\tau$ is the Denjoy-Wolff point of $\Phi_{t}$ for all $t>0$ and there exists $\beta \leq 0$ such that $\alpha_{\Phi_{t}}(\tau)=e^{\beta t}$.

If a semigroup ( $\Phi_{t}$ ) has no fixed points in $D$, we call the point $\tau \in \partial D$ given by Theorem 3.1 the Denjoy-Wolff point of the semigroup.

Definition 3.2. Let $D \subset \mathbb{C}^{n}$ be a bounded strongly convex domain with smooth boundary. Let $\left(\Phi_{t}\right)$ be a one-parameter semigroup of holomorphic self-maps of D. A point $p \in \partial D$ is called a boundary regular fixed point for $\left(\Phi_{t}\right)$, or a BRFP for short, if $p$ is a BRFP for $\Phi_{t}$ for all $t \geq 0$. The family of boundary dilatation coefficients of $\left(\Phi_{t}\right)$ will be denoted by $\left(\alpha_{t}(p)\right)$. A BRFP for $\left(\Phi_{t}\right)$ for which $\alpha_{t}(p) \leq 1$ for some $t>0$ is called a stationary point of the semigroup.

The boundary dilatation coefficients at BRFP's form a semigroup in $\left(\mathbb{R}_{0}^{+}, \cdot\right)$ :

Proposition 3.3. Let $D \subset \mathbb{C}^{n}$ be a bounded strongly convex domain with smooth boundary. Let $\left(\Phi_{t}\right)$ be a one-parameter semigroup of holomorphic self-maps of $D$. If $p \in \partial D$ is a BRFP for $\left(\Phi_{t}\right)$ then there exists $\beta \in \mathbb{R}$ such that $\alpha_{t}(p)=e^{\beta t}$ for all $t \geq 0$.

Proof. Let $\left(\varphi, \rho_{\varphi}, \widetilde{\rho}_{\varphi}\right)$ be the Lempert projection device associated with a complex geodesic such that $\varphi(1)=p$. Consider the following family of functions $T_{t}: D \rightarrow \mathbb{C}$ :

$$
T_{t}(z):=\frac{1-\tilde{\rho}_{\varphi} \circ \Phi_{t}(z)}{1-\widetilde{\rho}_{\varphi}(z)} .
$$

By Theorem 2.7 it follows that $\lim _{(0,1) \ni r \rightarrow 1} T_{t}(\gamma(r))=\alpha_{t}(p)$ for any curve $\gamma:(0,1) \rightarrow$ $D$ such that $\lim _{r \rightarrow 1} \gamma(r)=p$, the curve $\widetilde{\rho}_{\varphi}(\gamma(r))$ converges to 1 non-tangentially and $k_{D}\left(\gamma(r), \rho_{\varphi}(\gamma(r))\right) \rightarrow 0$ as $r \rightarrow 1$. By [8, Proposition 3.4], it follows that $[0,1) \ni r \mapsto$ $\Phi_{t}(\varphi(r))$ satisfies the same three properties which are satisfied by $\gamma$. Then for $s, t \geq 0$ we have

$$
T_{t+s}(\varphi(r))=\frac{1-\tilde{\rho}_{\varphi} \circ \Phi_{t}\left(\Phi_{s}(\varphi(r))\right)}{1-\widetilde{\rho}_{\varphi}\left(\Phi_{s}(\varphi(r))\right)} \cdot \frac{1-\widetilde{\rho}_{\varphi} \circ \Phi_{s}(\varphi(r))}{1-\widetilde{\rho}_{\varphi}(\varphi(r))}=T_{t}\left(\Phi_{s}(\varphi(r))\right) \cdot T_{s}(\varphi(r)),
$$

and taking the limit as $r \rightarrow 1$ it follows that $\alpha_{t+s}(p)=\alpha_{t}(p) \alpha_{s}(p)$. Since $\alpha_{t}(p)$ is clearly measurable in $t$, this concludes the proof. 
Later we will see how the number $\beta$ in Proposition 3.3 can be computed using the infinitesimal generator of the semigroup. Now we use the pluricomplex Green function to characterize vector fields which are infinitesimal generators. For this we need a lemma whose simple proof is left to the reader:

Lemma 3.4. Let $T>0$ be a positive real number and let $g:[0, T] \rightarrow \mathbb{R}$ be a function such that

(1) for all $a, b \in[0, T]$ and $\lambda \in[0,1]$ we have

$$
g(\lambda a+(1-\lambda) b) \leq \max \{g(a), g(b)\}
$$

(2) there exists the (right) derivative of $g$ at 0 and $g^{\prime}(0)>0$.

Then $g$ is increasing.

Now we can state and prove our characterizations of infinitesimal generators:

Theorem 3.5. Let $D \subset \subset \mathbb{C}^{n}$ be a strongly convex domain with smooth boundary. Let $F: D \rightarrow \mathbb{C}^{n}$ be holomorphic. The following are equivalent:

(1) The map $F$ is the infinitesimal generator of a semigroup of holomorphic self-maps of $D$.

(2) For all $z, w \in D$ with $z \neq w$,

$$
\left.d\left(k_{D}\right)\right|_{(z, w)} \cdot(F(z), F(w)) \leq 0 .
$$

(3) For all $z, w \in D$ with $z \neq w$,

$$
\left.d\left(G_{D}\right)\right|_{(z, w)} \cdot(F(z), F(w)) \leq 0 .
$$

(4) For each pair $z, w \in D$,

$$
k_{D}(z-r F(z), w-r F(w)) \geq k_{D}(z, w)
$$

for all $r>0$ such that $z-r F(z)$ and $w-r F(w)$ belong to $D$.

Proof. First of all we notice that by 1.2 we have $G_{D}(z, w)=\log \tanh k_{D}(z, w)$, and thus a simple computation shows that (2) and (3) are equivalent.

Next, we claim that (1) implies (3). Indeed, if $F$ is an infinitesimal generator in $D$ and $\left(\Phi_{t}\right)$ is the corresponding semigroup generated by $F$ then by $[1.1$, for all $z, w \in D$ with $z \neq w$ it follows that for all $t \geq 0$,

$$
G_{D}\left(\Phi_{t}(z), \Phi_{t}(w)\right)-G_{D}(z, w) \leq 0
$$

and it is equal to zero for $t=0$. Computing the incremental ratio in $t$ for $t=0$ we obtain 3.2.

Now, assume (2) holds. For $w \in D$, consider the Cauchy problem

$$
\left\{\begin{array}{l}
d \Phi / d t=F \circ \Phi \\
\Phi(0)=w
\end{array}\right.
$$


and denote by $\Phi_{w}:\left[0, \delta_{w}\right) \rightarrow D$ its maximal solution, for some $\delta_{w}>0$. To show that $F$ is an infinitesimal generator, it is enough to prove that $\delta_{w}=+\infty$ for all $w$.

To this end, let $z, w \in D$ with $z \neq w$ and let $\delta=\min \left\{\delta_{z}, \delta_{w}\right\}$. Let $g:[0, \delta) \ni t \mapsto$ $k_{D}\left(\Phi_{z}(t), \Phi_{w}(t)\right)$. By uniqueness of solutions of the above Cauchy problem, we know that $\Phi_{z}(t) \neq \Phi_{w}(t)$ for all $t \in[0, \delta)$. According to Lempert's work [28], [29] (see also [1. Proposition 2.6.40]), the function $g$ is smooth and differentiating with respect to $t$ we obtain, by (3.1),

$$
\begin{aligned}
g^{\prime}(t) & =\left.d\left(k_{D}\right)\right|_{\left(\Phi_{z}(t), \Phi_{w}(t)\right)} \cdot\left(\frac{d \Phi_{z}(t)}{d t}, \frac{d \Phi_{w}(t)}{d t}\right) \\
& =\left.d\left(k_{D}\right)\right|_{\left(\Phi_{z}(t), \Phi_{w}(t)\right)} \cdot\left(F\left(\Phi_{z}(t)\right), F\left(\Phi_{w}(t)\right)\right) \leq 0 .
\end{aligned}
$$

Therefore $g$ is non-increasing in $t$, so that

$$
k_{D}\left(\Phi_{z}(t), \Phi_{w}(t)\right) \leq k_{D}\left(\Phi_{z}(0), \Phi_{w}(0)\right)=k_{D}(z, w) .
$$

This implies that $\delta_{z}=\delta_{w}$, because if for instance $\delta_{z}<\delta_{w}$ then as $t \rightarrow \delta_{z}$ it would follow that $\Phi_{z}(t) \rightarrow \partial D$ while $\Phi_{w}(t) \rightarrow \Phi_{w}\left(\delta_{z}\right) \in D$, and then $k_{D}\left(\Phi_{z}(t), \Phi_{w}(t)\right) \rightarrow \infty$, contradicting (3.4).

By the arbitrariness of $z, w \in D$, this means that for all $z \in D$ we have $\delta_{z}=\delta$. Hence, by well known results on ODE's, we have a well defined analytic map $\Phi: D \times[0, \delta) \rightarrow D$ which is holomorphic in $z \in D$ and such that $\Phi(0, z)=z$ and $\partial \Phi / \partial t=F \circ \Phi$. Also, $\Phi(t+s, z)=\Phi(t, \Phi(s, z))$ for all $s, t \geq 0$ such that $s+t<\delta$ and $z \in D$. This implies that $\delta=+\infty$. Indeed, if $\delta<+\infty$, let $2 \delta>t>\delta$ and let $s>0$ be such that $t-s<\delta$, $s<\delta$. Define $\Phi_{z}(t):=\Phi(t-s, \Phi(s, z))$. This is well defined and solves the Cauchy problem for $z$, contrary to the maximality of $\delta$.

Thus we have proved that (1), (2) and (3) are equivalent.

Now, let us prove that (4) implies (2). Let $z, w \in D, z \neq w$, and $r>0$ be such that $z-r F(z)$ and $w-r F(w)$ belong to $D$. By convexity, $z-t F(z)$ and $w-t F(w)$ belong to $D$ for all $t \in[0, r]$. Therefore, the function $g:[0, r] \rightarrow \mathbb{R}$ given by

$$
g(t)=k_{D}(z-t F(z), w-t F(w))
$$

is well defined and, again by Lempert's result, since $z \neq w$, it is differentiable at 0 . By hypothesis, $g(t) \geq g(0)$ for all $t \geq 0$. Therefore $g^{\prime}(0) \geq 0$. But

$$
g^{\prime}(0)=\left.d\left(k_{D}\right)\right|_{(z, w)} \cdot(-F(z),-F(w))=-\left.d\left(k_{D}\right)\right|_{(z, w)} \cdot(F(z), F(w)) .
$$

Thus, $\left.d\left(k_{D}\right)\right|_{(z, w)} \cdot(F(z), F(w)) \leq 0$, and (2) holds.

In order to finish the proof we show that (2) implies (4). We consider the following two possible cases:

(I) $\left.d\left(k_{D}\right)\right|_{(z, w)} \cdot(F(z), F(w))<0$.

(II) $\left.d\left(k_{D}\right)\right|_{(z, w)} \cdot(F(z), F(w))=0$. 
Case (I). Fix $r>0$ such that $z-r F(z)$ and $w-r F(w)$ belong to $D$. Then $z-t F(z)$ and $w-t F(w)$ belong to $D$ for all $t \in[0, r]$. Therefore, the function $g:[0, r] \rightarrow \mathbb{R}$ given by

$$
g(t)=k_{D}(z-t F(z), w-t F(w))
$$

is well defined and, since $z \neq w$, it is differentiable at 0 with $g^{\prime}(0)=\left.d\left(k_{D}\right)\right|_{(z, w)}$. $(-F(z),-F(w))>0$. Moreover, by [32, Proposition 3.8], given $z_{1}, z_{2}, w_{1}, w_{2} \in D$ and $\lambda \in[0,1]$, we have

$$
k_{D}\left(\lambda z_{1}+(1-\lambda) z_{2}, \lambda w_{1}+(1-\lambda) w_{2}\right) \leq \max \left\{k_{D}\left(z_{1}, w_{1}\right), k_{D}\left(z_{2}, w_{2}\right)\right\} .
$$

In particular, if $a, b \in[0, r]$ and $\lambda \in[0,1]$, then

$$
\begin{aligned}
g(\lambda & +(1-\lambda) b) \\
& =k_{D}(z-(\lambda a+(1-\lambda) b) F(z), w-(\lambda a+(1-\lambda) b) F(w)) \\
& =k_{D}(\lambda(z-a F(z))+(1-\lambda)(z-b F(z)), \lambda(w-a F(w))+(1-\lambda)(w-b F(w))) \\
& \leq \max \left\{k_{D}(z-a F(z), w-a F(w)), k_{D}(z-b F(z), w-b F(w))\right\} \\
& =\max \{g(a), g(b)\} .
\end{aligned}
$$

Therefore, $g$ satisfies the hypothesis of Lemma 3.4 and thus it is non-decreasing, so that

$$
k_{D}(z-t F(z), w-t F(w)) \geq k_{D}(z, w) \quad \text { for all } t \in[0, r] .
$$

Case (II). Let $G: D \rightarrow \mathbb{C}^{n}$ holomorphic be an infinitesimal generator in $D$ such that $\left.d\left(k_{D}\right)\right|_{(z, w)} \cdot(G(z), G(w))<0$. Such a map can be constructed as follows. Up to translations we can assume that $z=O$, the origin in $\mathbb{C}^{n}$. Let $a<0$. By convexity, the family of functions $\Phi_{t}: z \mapsto e^{a t} z$ is a semigroup of holomorphic self-maps of $D$. The associated infinitesimal generator is $G(z)=a z$. Therefore

$$
\left.d\left(k_{D}\right)\right|_{(O, w)} \cdot(G(O), G(w))=\left.\operatorname{ad}\left(k_{D}\right)\right|_{(O, w)} \cdot(O, w) .
$$

Now, the vector $(O, w)$ points outward with respect to the boundary of the Kobayashi ball of center $O$ and radius $k_{D}(O, w)$ because Kobayashi balls of convex domains are convex (see, e.g., [1, Proposition 2.3.46]). Since Kobayashi balls are level sets of $k_{D}$, this implies that $\left.d\left(k_{D}\right)\right|_{(O, w)} \cdot(O, w) \neq 0$. Hence $\left.d\left(k_{D}\right)\right|_{(O, w)} \cdot(G(O), G(w)) \neq 0$ and, by the already proved equivalence between (1) and (2), actually $\left.d\left(k_{D}\right)\right|_{(z, w)} \cdot(G(z), G(w))<0$.

Now fix $\epsilon>0$ and consider the vector field $H:=F+\epsilon G$. This is an infinitesimal generator of a semigroup of holomorphic self-maps in $D$ (because $F+\epsilon G$ satisfies (3.1) and by the equivalence between (1) and (2)). Now, by construction, $\left.d\left(k_{D}\right)\right|_{(z, w)}$. $(H(z), H(w))<0$ and, by what we proved in Case (I), $k_{D}(z-r H(z), w-r H(w)) \geq$ $k_{D}(z, w)$ for all $r>0$ such that $z-r H(z), w-r H(w) \in D$. Now, letting $\epsilon$ tend to 0 we end the proof.

As a corollary we have the following characterization of groups of biholomorphisms of $D$ :

Corollary 3.6. Let $D \subset \subset \mathbb{C}^{n}$ be a strongly convex domain with smooth boundary. Let $F: D \rightarrow \mathbb{C}^{n}$ be holomorphic. The following are equivalent: 
(1) The map $F$ is the infinitesimal generator of a group of holomorphic self-maps of $D$.

(2) For all $z, w \in D$ with $z \neq w$,

$$
\left.d\left(k_{D}\right)\right|_{(z, w)} \cdot(F(z), F(w))=0 .
$$

(3) For all $z, w \in D$ with $z \neq w$,

$$
\left.d\left(G_{D}\right)\right|_{(z, w)} \cdot(F(z), F(w))=0 .
$$

Proof. Apply Theorem 3.5 to $F$ and $-F$.

Remark 3.7. In case $D=\mathbb{B}^{n}$, using $(1.3)$, inequality $(3.2)$ assumes a simple expression given by

$$
\frac{\operatorname{Re}\langle z, F(z)\rangle}{1-\|z\|^{2}}+\frac{\operatorname{Re}\langle w, F(w)\rangle}{1-\|w\|^{2}} \leq \operatorname{Re} \frac{\langle F(z), w\rangle+\langle z, F(w)\rangle}{1-\langle z, w\rangle} .
$$

In fact, in case $D=\mathbb{B}^{n}$, Theorem 3.5 with (3.5) replacing (3.2) was proven with different methods by Reich and Shoikhet [31, Theorem 2.1] (see also [20], [27]).

For boundary regular fixed points, we have the following result:

Theorem 3.8. Let $D \subset \subset \mathbb{C}^{n}$ be a strongly convex domain with smooth boundary. Let $F: D \rightarrow \mathbb{C}^{n}$ be the holomorphic infinitesimal generator of a semigroup $\left(\Phi_{t}\right), \beta \in \mathbb{R}$ and $p \in \partial D$. The following are equivalent:

(1) The semigroup $\left(\Phi_{t}\right)$ has a BRFP at $p$ with boundary dilatation coefficients $\alpha_{t}(p) \leq$ $e^{\beta t}$ for all $t \geq 0$.

(2) $d\left(u_{D, p}\right)_{z} \cdot \overline{F(z)}+\beta u_{D, p}(z) \leq 0$ for all $z \in D$.

Moreover, if $p$ is a BRFP for $\left(\Phi_{t}\right)$ then the boundary dilatation coefficient of $\Phi_{t}$ is $\alpha_{t}(p)=e^{-t b}$ with $b=\inf _{z \in D} d\left(u_{D, p}\right)_{z} \cdot F(z) / u_{D, p}(z)$.

Proof. Suppose (1) holds. Then $u_{D, p}\left(\Phi_{t}(z)\right)-e^{-t \beta} u_{D, p}(z) \leq 0$ for all $t \geq 0$ and $z \in D$. In particular,

$$
\begin{aligned}
0 & \geq \lim _{t \rightarrow 0^{+}} \frac{u_{D, p}\left(\Phi_{t}(z)\right)-e^{-t \beta} u_{D, p}(z)}{t} \\
& =\left.\frac{\partial}{\partial t}\left[u_{D, p}\left(\Phi_{t}(z)\right)-e^{-t \beta} u_{D, p}(z)\right]\right|_{t=0}=d\left(u_{D, p}\right)_{z} \cdot F(z)+\beta u_{D, p}(z),
\end{aligned}
$$

and (2) follows.

Conversely, assume (2) holds. Fix $z \in D$ and let $g(t):=u_{D, p}\left(\Phi_{t}(z)\right)-e^{-t \beta} u_{D, p}(z)$. We have to show that $g(t) \leq 0$ for all $t \geq 0$. Differentiating $g$, we obtain

$$
\begin{aligned}
g^{\prime}(t) & =d\left(u_{D, p}\right)_{\Phi_{t}(z)} \cdot \frac{\partial \Phi_{t}}{\partial t}(z)+\beta e^{-\beta t} u_{D, p}(z) \\
& =d\left(u_{D, p}\right)_{\Phi_{t}(z)} \cdot F\left(\Phi_{t}(z)\right)+\beta e^{-\beta t} u_{D, p}(z) \\
& =d\left(u_{D, p}\right)_{\Phi_{t}(z)} \cdot F\left(\Phi_{t}(z)\right)+\beta u_{D, p}\left(\Phi_{t}(z)\right)-\beta g(t) .
\end{aligned}
$$


Therefore, using hypothesis (2) we see that for all $t \geq 0$,

$$
g^{\prime}(t)+\beta g(t) \leq 0
$$

Now let $h(t):=-\left(g^{\prime}(t)+\beta g(t)\right) \geq 0$. Solving the differential equation $g^{\prime}(t)+\beta g(t)+$ $h(t)=0$ with initial value $g(0)=0$, we obtain

$$
g(t)=-e^{-\beta t} \int_{0}^{t} e^{\beta s} h(s) d s \leq 0,
$$

and thus (1) follows.

Finally, the last statement comes directly from Proposition 3.3 and the equivalence between (1) and (2).

Remark 3.9. If $\beta \leq 0$ in Theorem 3.8, it follows that for all $z \in D$ the function $[0,+\infty) \ni t \mapsto u_{D, p}\left(\Phi_{t}(z)\right)-e^{-t \beta} u_{D, p}(z)$ is decreasing. Indeed, for $s>t$, we have

$$
\begin{aligned}
u_{D, p}\left(\Phi_{s}(z)\right)-e^{-s \beta} u_{D, p}(z) & =u_{D, p}\left(\Phi_{s-t}\left(\varphi_{t}(z)\right)\right)-e^{-s \beta} u_{D, p}(z) \\
& \leq e^{-(s-t) \beta}\left[u_{D, p}\left(\Phi_{t}(z)\right)-e^{-t \beta} u_{D, p}(z)\right] \\
& \leq u_{D, p}\left(\Phi_{t}(z)\right)-e^{-t \beta} u_{D, p}(z) .
\end{aligned}
$$

We end up this section with a Berkson-Porta like characterization of infinitesimal generators.

Definition 3.10. Let $D \subset \subset \mathbb{C}^{n}$ be a strongly convex domain with smooth boundary, $F: D \rightarrow \mathbb{C}^{n}$ holomorphic and $p \in \partial D$. We say that $F \in C_{E}^{1}(p)$ if for any horosphere $E_{D}(p, R)$ there exists an $(n \times n)$-matrix A such that

$$
\lim _{E_{D}(p, R) \ni z \rightarrow p} d F_{z}=A .
$$

Theorem 3.11. Let $D \subset \subset \mathbb{C}^{n}$ be a strongly convex domain with smooth boundary. Let $p \in \partial D, F: D \rightarrow \mathbb{C}^{n}$ holomorphic and assume that $F \in C_{E}^{1}(p)$. Then $F$ is the infinitesimal generator of a semigroup of holomorphic self-maps of $D$ with a stationary point at $p$ if and only if

$$
d\left(u_{D, p}\right)_{z} \cdot F(z) \leq 0 \quad \text { for all } z \in D .
$$

Proof. One direction follows directly from Theorem 3.8 .

Conversely, assume that (3.7) holds. Fix $w_{0} \in D$ and let $\gamma:[0, \delta) \rightarrow D$ be the maximal solution of the Cauchy problem

$$
\left\{\begin{array}{l}
d \gamma / d t=F \circ \gamma \\
\gamma(0)=w_{0}
\end{array}\right.
$$

It is enough to prove $\delta=+\infty$. Assume by contradiction that $\delta<+\infty$. Let $g(t):=$ $u_{D, p}(\gamma(t))$ for $t \in[0, \delta)$. Differentiating $g$, we obtain, by (3.7),

$$
g^{\prime}(t)=d\left(u_{D, p}\right)_{\gamma(t)}\left(\gamma^{\prime}(t)\right)=d\left(u_{D, p}\right)_{\gamma(t)}(F(\gamma(t))) \leq 0 .
$$


Thus $u_{D, p}(\gamma(t)) \leq u_{D, p}(\gamma(0))=u_{D, p}\left(w_{0}\right)$ for all $t \in[0, \delta)$. This means that if $w_{0} \in E_{D}(p, R)$ then $\gamma(t)$ belongs to $E_{D}(p, R)$ for all $t \in[0, \delta)$. In particular, since $\overline{E_{D}(p, R)} \cap \partial D=\{p\}$, it means that $\lim _{t \rightarrow \delta} \gamma(t)=p$.

Since $F \in C_{E}^{1}(p)$ and $\partial E_{D}(p, R)$ is Lipschitz (it is actually $C^{1,1}$ at $p$ and smooth elsewhere, see [14, Section 4]) by (a very simple form of) the Whitney extension theorem there exists a function $\tilde{F}: \mathbb{C}^{n} \rightarrow \mathbb{C}^{n}$ of class $C^{1}$ such that $\left.\tilde{F}\right|_{E_{D}(p, R)}=F$. If $\tilde{F}(p)=0$ then the Cauchy problem

$$
\left\{\begin{array}{l}
d \eta / d t=\tilde{F} \circ \eta, \\
\eta(\delta)=p
\end{array}\right.
$$

has the unique solution $\eta(t) \equiv p$. In particular, $\gamma$ cannot reach $p$ in a finite time, which contradicts $\delta<+\infty$.

To conclude the proof we are left to show that necessarily $\tilde{F}(p)=0$. But this follows at once from the fact that for any $z \in E_{D}(p, R)$ the solution of the Cauchy problem

$$
\left\{\begin{array}{l}
d \gamma^{z} / d t=\tilde{F} \circ \gamma^{z}, \\
\gamma^{z}(0)=z
\end{array}\right.
$$

is such that $\gamma^{z}(t) \in D$ for $t \in\left[0, \delta^{z}\right)$ for a suitable $\delta^{z} \in(0,+\infty]$ and, arguing as for $\gamma$, $\lim _{t \rightarrow \delta^{z}} \gamma^{z}(t)=p$.

Remark 3.12. If $D=\mathbb{D}$, the unit disc in $\mathbb{C}$, then the conclusion of Theorem 3.11 holds without any regularity assumption on $F$ at $p \in \partial \mathbb{D}$. Indeed, a direct computation shows that (3.7) reduces exactly to the Berkson-Porta formula [6].

If $D=\mathbb{B}^{n}$, the unit ball of $\mathbb{C}^{n}$, a direct computation shows that 3.7$)$ corresponds to

$$
\frac{\operatorname{Re}\langle F(z), z\rangle}{1-\left\|z^{2}\right\|} \leq \operatorname{Re} \frac{\langle F(z), p\rangle}{1-\langle z, p\rangle} .
$$

In fact, for $D=\mathbb{B}^{n}$ and with the additional hypothesis that $F$ extends holomorphically through $\partial \mathbb{B}^{n}$, Theorem 3.11 follows from [3, Theorem 3.1].

\section{Boundary behavior of infinitesimal generators}

In all this section, $D$ denotes a bounded strongly convex domain in $\mathbb{C}^{n}$ with smooth boundary.

Before proving the main result of this section, we examine two significant examples. We will use a lemma whose proof can be derived from the proof of [12, Theorem 1.4].

Lemma 4.1. Let $a, b \in \mathbb{C}^{n}$ and $A \in \mathbb{C}^{n \times n}$, and

$$
G(z)=a-\langle z, a\rangle z-[A z+\langle z, b\rangle z] .
$$

Then $G$ is the infinitesimal generator of a continuous semigroup of holomorphic self-maps of $\mathbb{B}^{n}$ if and only if

$$
|\langle b, u\rangle| \leq \operatorname{Re}\langle A u, u\rangle \quad \text { for all } u \in \partial \mathbb{B}^{n} .
$$

Moreover, if equality holds at every point of $\partial \mathbb{B}^{n}$, then $G$ is the infinitesimal generator of a continuous group of holomorphic self-maps of $\mathbb{B}^{n}$. 
Example 4.2. Let us consider $F: \mathbb{B}^{2} \rightarrow \mathbb{C}^{2}$ given by $F\left(z_{1}, z_{2}\right)=\left(0,-z_{2} /\left(1-z_{1}\right)\right)$. Let $e_{1}=(1,0)$. By a direct computation one can see that $F$ is the infinitesimal generator of a semigroup $\left(\Phi_{t}\right)$ of holomorphic self-maps of $\mathbb{B}^{2}$ which pointwise fixes the slice $\mathbb{D} \ni$ $\zeta \mapsto(\zeta, 0)$. Clearly $d\left(u_{\mathbb{B}^{2}, e_{1}}\right)_{z} \circ F(z) \leq 0$ for all $z \in \mathbb{B}^{2}$. Thus $F$ has a stationary point at

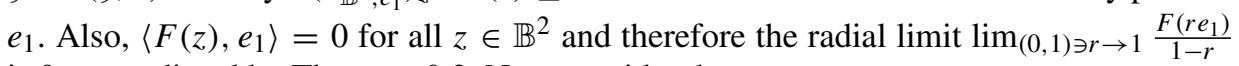
is 0 , as predicted by Theorem 0.3 . Now consider the map

$$
\eta\left(z_{1}, z_{2}\right)=\left(\frac{-s z_{2}+(1-\beta) z_{1}+\beta}{-s z_{2}-\beta z_{1}+1+\beta}, \frac{z_{2}+s z_{1}-s}{-s z_{2}-\beta z_{1}+1+\beta}\right)
$$

where $\operatorname{Re} \beta>0$ and $s=\sqrt{2 \operatorname{Re} \beta}$. Notice that $\eta: \mathbb{B}^{2} \rightarrow \mathbb{B}^{2}$ is a parabolic automorphism such that $\eta\left(e_{1}\right)=e_{1}$ (see [7, Example 5.1]). Hence $\tilde{F}\left(z_{1}, z_{2}\right):=d \eta_{\eta\left(z_{1}, z_{2}\right)}^{-1} \cdot F\left(\eta\left(z_{1}, z_{2}\right)\right.$ ) is the infinitesimal generator of the semigroup $\left(\tilde{\Phi}_{t}\right)$ of holomorphic self-maps of $\mathbb{B}^{2}$, where $\tilde{\Phi}_{t}=\eta^{-1} \circ \Phi_{t} \circ \eta$. Thus $\tilde{\Phi}_{t}$ pointwise fixes the slice $\eta^{-1}(\zeta, 0)$ for $\zeta \in \mathbb{D}$. A direct computation shows that $F(\eta(r, 0))=(0, s)$ for $r \in(0,1)$. Thus, since

$$
d \eta_{r e_{1}}=\frac{1}{(-r \beta+1+\beta)^{2}}\left(\begin{array}{cc}
1 & s(r-1) \\
s & \bar{\beta}(r-1)+1
\end{array}\right)
$$

it follows that $\tilde{F}(r, 0)=(\beta(1-r)+1)\left(-s^{2}(r-1), s\right)$ for $r \in(0,1)$. In particular, the radial limit of $\tilde{F}$ at $e_{1}$ is not zero. A direct computation shows that

$$
\lim _{(0,1) \ni r \rightarrow 1} \frac{\tilde{F}_{1}\left(r e_{1}\right)}{r-1}=-s^{2}<0 .
$$

Hence the semigroup of $\mathbb{D}$ generated by the "projection" $\tilde{F}_{1}\left(\zeta e_{1}\right)$ of $\tilde{F}$ to the slice $\mathbb{D} \ni$ $\zeta \mapsto \zeta e_{1}$ has Denjoy-Wolff point at 1 , with boundary dilatation coefficients $\left(e^{-s^{2} t}\right)$. However, by construction, $e_{1}$ is a stationary point for the semigroup $\left(\tilde{\Phi}_{t}\right)$, with boundary dilatation coefficients all equal to 1 .

Example 4.3. Let $G: \mathbb{B}^{2} \rightarrow \mathbb{C}^{2}$ be the map defined by $G\left(z_{1}, z_{2}\right)=\left(\frac{-1}{3} i z_{1}, i z_{2}\right)$. The map $G$ is the infinitesimal generator of a (semi)group $\left(\Phi_{t}\right)$ of elliptic automorphisms fixing the origin (see Lemma 4.1). Let us consider the automorphism of $\mathbb{B}^{2}$ given by

$$
\psi\left(z_{1}, z_{2}\right)=\left(\frac{-\sqrt{3} z_{1}}{2-z_{2}}, \frac{1-2 z_{2}}{2-z_{2}}\right)
$$

(see [1, Lemma 2.2.1]). We note that $\psi=\psi^{-1}$. Such an automorphism maps the slice $\left\{\left(z_{1}, z_{2}\right) \in \mathbb{B}^{2}: z_{2}=0\right\}$ to the slice $\left\{\left(z_{1}, z_{2}\right) \in \mathbb{B}^{2}: z_{2}=1 / 2\right\}$. Define $P\left(z_{1}, z_{2}\right):=$ $d \psi_{\psi^{-1}\left(z_{1}, z_{2}\right)} \cdot G\left(\psi^{-1}\left(z_{1}, z_{2}\right)\right)$. A direct computation shows that

$$
P\left(z_{1}, z_{2}\right)=\left(\frac{-2 i}{3} z_{1} z_{2}, \frac{-i}{3}\left(z_{2}-2\right)\left(2 z_{2}-1\right)\right) .
$$

Let $\left(\Phi_{t}^{P}\right)$ be the group of automorphisms generated by $P$. It is obtained by conjugation from the group $\left(\Phi_{t}\right)$ and therefore has only one fixed point. Since $P\left(z_{1}, 0\right)=\left(0, \frac{-2 i}{3}\right)$ 
(and $P$ is holomorphic past the boundary) it follows that $\left(\Phi_{t}^{P}\right)$ cannot have a BRFP at any point of the boundary of the slice $\left\{\left(z_{1}, z_{2}\right) \in \mathbb{B}^{2}: z_{2}=0\right\}$. However, obviously

$$
\lim _{\mathbb{D} \ni \zeta \rightarrow 1} \frac{P_{1}(\zeta, 0)}{\zeta-1}=0 .
$$

Finally, let

$$
H\left(z_{1}, z_{2}\right)=\left(\frac{2 i}{3} z_{2}\left(z_{1}-1\right), \frac{2 i}{3}\left(1+z_{2}^{2}-z_{1}\right)\right) .
$$

The map $H: \mathbb{B}^{2} \rightarrow \mathbb{C}^{2}$ is the infinitesimal generator of a group of automorphisms (see again Lemma 4.1] with the property that $H\left(e_{1}\right)=(0,0)$. Define $F=P+H$. Then $F$ is the infinitesimal generator of a group of automorphisms and a direct computation shows that

$$
F\left(z_{1}, z_{2}\right)=\left(-\frac{2 i}{3} z_{2},-\frac{5 i}{3} z_{2}-\frac{2 i}{3} z_{1}\right) .
$$

Thus the group generated by $F$ has a unique fixed point at $(0,0)$ and no BRFPs on $\partial \mathbb{B}^{2}$. However, the semigroup generated by $\mathbb{D} \ni \zeta \mapsto F_{1}(\zeta, 0)$ on $\mathbb{D}$ is trivial and

$$
\lim _{\mathbb{D} \ni \zeta \rightarrow e^{i \theta}} \frac{F_{1}(\zeta, 0)}{\zeta-1}=0
$$

for all $\theta \in \mathbb{R}$. Notice that the slice $\left\{\left(z_{1}, z_{2}\right) \in \mathbb{B}^{2}: z_{2}=0\right\}$ contains the fixed point of the semigroup.

The previous two examples show that, on the one hand, the requirement that the radial limit exists in Theorem 0.3 is sufficient but not necessary for the existence of BRFPs. Also, even if a BRFP exists, say at $p \in \partial \mathbb{B}^{n}$, the radial limit of the incremental ratio of the projection of the infinitesimal generator along a complex geodesic containing $p$ might not give information on the boundary dilatation coefficients of the semigroup at $p$. On the other hand, the sole information on the existence of the limit of the incremental ratio along a given complex geodesic containing the point $p \in \partial \mathbb{B}^{n}$ does not imply existence of a BRFP at $p$. Last but not least, an unexpected phenomenon takes place for infinitesimal generators: the behavior of the semigroup generated by the "restriction" of the infinitesimal generator to one complex geodesic-even a complex geodesic containing fixed points of the semigroup - can be completely different from the behavior of the semigroup in the ball (cf. Theorem 2.7).

Definition 4.4. Let $F: D \rightarrow \mathbb{C}^{n}$ be a holomorphic infinitesimal generator. For a Lempert projection device $\left(\varphi, \rho_{\varphi}, \widetilde{\rho}_{\varphi}\right)$ we set $f_{\varphi}(\zeta):=d\left(\widetilde{\rho}_{\varphi}\right)_{\varphi(\zeta)} \cdot F(\varphi(\zeta))$, a holomorphic vector field on $\mathbb{D}$.

Proposition 4.5. Let $F: D \rightarrow \mathbb{C}^{n}$ be a holomorphic infinitesimal generator. Let $\left(\varphi, \rho_{\varphi}, \widetilde{\rho}_{\varphi}\right)$ be a Lempert projection device with $p=\varphi(1) \in \partial D$. Then the vector field $f_{\varphi}(\zeta)$ is a holomorphic infinitesimal generator in $\mathbb{D}$. Moreover, if there exists $\beta \in \mathbb{R}$ such that $d\left(u_{D, p}\right)_{z} \cdot F(z)+\beta u_{D, p}(z) \leq 0$ for all $z \in D$, then $d\left(u_{\mathbb{D}, 1}\right)_{\zeta} \cdot f_{\varphi}(\zeta)+\beta u_{\mathbb{D}, 1}(\zeta) \leq 0$ for all $\zeta \in \mathbb{D}$. 
Proof. Consider the pluricomplex Green function $G_{D}: D \times D \rightarrow \mathbb{R}$. Its differential $d G_{D}: T D \times T D \rightarrow T \mathbb{R}$ can be decomposed as $d G_{D}=d_{z} G_{D}+d_{w} G_{D}$ where $d G_{D}(u, v)=d_{z} G_{D}(u)+d_{w} G_{D}(v)$ for $(u, v) \in T D \times T D$. With this notation, Theorem 3.5 implies that for all $z \neq w$,

$$
d\left(G_{D}\right)_{(z, w)} \cdot(F(z), F(w))=\left.d_{z}\left(G_{D}\right)\right|_{(z, w)} \cdot F(z)+\left.d_{w}\left(G_{D}\right)\right|_{(z, w)} \cdot F(w) \leq 0 .
$$

Now let $z=\varphi(\eta)$ and $w=\varphi(\zeta)$ for $\eta \neq \zeta \in \mathbb{D}$. We claim that

$$
\left.d_{w}\left(G_{D}\right)\right|_{(\varphi(\eta), \varphi(\zeta))} \cdot F(\varphi(\zeta))=\left.d_{w}\left(G_{D}\right)\right|_{(\varphi(\eta), \varphi(\zeta))}\left(d\left(\rho_{\varphi}\right)_{\varphi(\zeta)} \cdot F(\varphi(\zeta))\right) .
$$

Assume that 4.2 is true. According to 1.5 we also have $G_{D}(\varphi(\eta), \varphi(\zeta))=G_{\mathbb{D}}(\eta, \zeta)$ for all $\zeta \in \mathbb{D}$, thus by 4.2 ,

$$
\begin{aligned}
\left.d_{w}\left(G_{\mathbb{D}}\right)\right|_{(\eta, \zeta)}\left(f_{\varphi}(\zeta)\right) & =\left.d_{w}\left(G_{D}\right)\right|_{(\varphi(\eta), \varphi(\zeta))} \cdot\left(d \varphi_{\zeta}\left(f_{\varphi}(\zeta)\right)\right) \\
& =\left.d_{w}\left(G_{D}\right)\right|_{(\varphi(\eta), \varphi(\zeta))} \cdot\left(d\left(\rho_{\varphi}\right)_{\varphi(\zeta)}(F(\varphi(\zeta)))\right) \\
& =\left.d_{w}\left(G_{D}\right)\right|_{(\varphi(\eta), \varphi(\zeta))} \cdot F(\varphi(\zeta)) .
\end{aligned}
$$

A similar equation holds for $\left.\left.d_{z}\left(G_{D}\right)\right|_{(z, w)}\right|_{(\varphi(\eta), \varphi(\zeta))} \cdot F(\varphi(\eta))$, by swapping the roles of $\eta$ and $\zeta$ in the previous argument. Thus

$$
\left.\left.d\left(G_{\mathbb{D}}\right)\right|_{(\eta, \zeta)} \cdot\left(f_{\varphi}(\eta), f_{\varphi}(\zeta)\right)=\left.d\left(G_{D}\right)\right|_{(\varphi(\eta), \varphi(\zeta))} \cdot(F(\varphi(\eta)), F(\varphi(\zeta)))\right) \leq 0
$$

for all $\zeta, \eta \in \mathbb{D}$ with $\zeta \neq \eta$, which implies that $f_{\varphi}$ is an infinitesimal generator on $\mathbb{D}$ by Theorem 3.5

Now it remains to prove claim 4.2). Since $\rho_{\varphi}$ is holomorphic, we have $d \rho_{\varphi}=\partial \rho_{\varphi}$ and the Lempert projection $\rho_{\varphi}$ determines a holomorphic splitting of the exact sequence of holomorphic bundles

$$
\left.0 \rightarrow T \varphi(\mathbb{D}) \stackrel{\iota}{\rightarrow} T D\right|_{\varphi(\mathbb{D})} \rightarrow N_{\varphi(\mathbb{D}), D} \rightarrow 0
$$

given by

$$
T_{\varphi(\zeta)} D=\iota\left(d \rho_{\varphi}\left(T_{\varphi(\zeta)} D\right)\right) \oplus \operatorname{Ker} d\left(\rho_{\varphi}\right)_{\varphi(\zeta)}
$$

Now let $B_{D}(\varphi(0), R)=\left\{z \in D: k_{D}(z, \varphi(0))<R\right\}$ be a Kobayashi ball for $D$ and let $\zeta_{1} \in \mathbb{D}$ be such that $\varphi\left(\zeta_{1}\right) \in \partial B_{D}(\varphi(0), R)$. It is known-and can be easily proven using Lempert's special coordinates (see [28], [29]) — that

$$
T_{\varphi\left(\zeta_{1}\right)}^{\mathbb{C}} \partial B_{D}(\varphi(0), R)=\operatorname{Ker} d\left(\rho_{\varphi}\right)_{\varphi\left(\zeta_{1}\right)}
$$

where, as usual, $T_{\varphi\left(\zeta_{1}\right)}^{\mathbb{C}} \partial B_{D}(\varphi(0), R)$ denotes the complex tangent space of $\partial B_{D}(\varphi(0), R)$ at $\varphi\left(\zeta_{1}\right)$. By 1.2 it follows that

$$
\partial B_{D}(\varphi(0), R)=\left\{z \in D: G_{D}(\varphi(0), z)=r\right\}
$$

for a suitable $r<0$, and in particular

$$
T_{\varphi\left(\zeta_{1}\right)} \partial B_{D}(\varphi(0), R)=\operatorname{Ker}\left(d_{w} G_{D}\right)_{\left(\varphi(0), \varphi\left(\zeta_{1}\right)\right)} .
$$


Now, consider the Kobayashi ball $B_{D}(\varphi(\eta), R)$ with $R=R(\varphi(\zeta))>0$ such that $\varphi(\zeta) \in \partial B_{D}(\varphi(\eta), R)$. Equations 4.3 and 4.4 yield

$$
\operatorname{Ker} d\left(\rho_{\varphi}\right)_{\varphi(\zeta)}=T_{\varphi(\zeta)}^{\mathbb{C}} \partial B_{D}(\varphi(\eta), R) \subset T_{\varphi(\zeta)} \partial B_{D}(\varphi(\eta), R)=\operatorname{Ker}\left(d_{w} G_{D}\right)_{(\varphi(\eta), \varphi(\zeta))},
$$

from which equation (4.2) follows, and the claim is proved.

In order to prove the last assertion of the proposition, we argue much as before. Let $\zeta \in \mathbb{D}$ and let $E_{D}(\varphi(1), R)$ be the horosphere in $D$ which contains $\varphi(\zeta)$ on its boundary. Then (again using Lempert's special coordinates, see [13, p. 517])

$$
T_{\varphi\left(\zeta_{1}\right)}^{\mathbb{C}} \partial E_{D}(\varphi(1), R)=\operatorname{Ker} d\left(\rho_{\varphi}\right)_{\varphi\left(\zeta_{1}\right)}
$$

and

$$
T_{\varphi\left(\zeta_{1}\right)} \partial E_{D}(\varphi(1), R)=\operatorname{Ker} d\left(u_{D, \varphi(1)}\right)_{\varphi\left(\zeta_{1}\right)} .
$$

Since $T_{\varphi(\zeta)}^{\mathbb{C}} \partial E_{D}(\varphi(1), R) \subset T_{\varphi(\zeta)} \partial E_{D}(\varphi(1), R)$, equation 4.5 yields

$$
d\left(u_{D, \varphi(1)}\right)_{\varphi(\zeta)} \cdot F(\varphi(\zeta))=d\left(u_{D, \varphi(1)}\right)_{\varphi(\zeta)}\left(d\left(\rho_{\varphi}\right)_{\varphi(\zeta)} \cdot F(\varphi(\zeta))\right),
$$

and since $u_{D, \varphi(1)} \circ \varphi(\zeta)=a_{\varphi} u_{\mathbb{D}, 1}(\zeta)$ for some $a_{\varphi}>0$ by (1.6), we have

$$
d\left(u_{\mathbb{D}, 1}\right)_{\zeta} \cdot f_{\varphi}(\zeta)+\beta u_{\mathbb{D}, 1}(\zeta)=\frac{1}{a_{\varphi}}\left[d\left(u_{D, p}\right)_{\varphi(\zeta)} \cdot F(\varphi(\zeta))+\beta u_{D, p}(\varphi(\zeta))\right]
$$

If $d\left(u_{D, p}\right)_{z} \cdot F(z)+\beta u_{D, p}(z) \leq 0$ for all $z \in D$ then $d\left(u_{\mathbb{D}, 1}\right)_{\zeta} \cdot f_{\varphi}(\zeta)+\beta u_{\mathbb{D}, 1}(\zeta) \leq 0$ for all $\zeta \in \mathbb{D}$ as stated.

Before stating and proving the main result of this section we need a preliminary lemma.

Lemma 4.6. Let $\mathbb{D} \subset \mathbb{C}$ be the unit disc in $\mathbb{C}$. Let $G$ be the infinitesimal generator of a semigroup $\left(\eta_{t}\right)$ of holomorphic self-maps of $\mathbb{D}$. The following are equivalent:

(1) The point 1 is a boundary regular fixed point for $\left(\eta_{t}\right)$.

(2) There exists $C>0$ such that the radial limit satisfies

$$
\limsup _{(0,1) \ni r \rightarrow 1} \frac{|G(r)|}{1-r} \leq C \text {. }
$$

Moreover, if 1 is a BRFP for $\left(\eta_{t}\right)$ with boundary dilatation coefficients $\alpha_{t}(1)=e^{b t}$ then

$$
\angle \lim _{\zeta \rightarrow 1} \frac{G(\zeta)}{\zeta-1}=b
$$


Proof. If (1) holds then the result follows directly from [15, Theorem 1].

Conversely, hypothesis (2) implies that $\lim _{r \rightarrow 1} G(r)=0$. By Berkson-Porta's Theorem 0.1 there exists a point $b \in \overline{\mathbb{D}}$ and a holomorphic function $p: \mathbb{D} \rightarrow \mathbb{C}$ with $\operatorname{Re} p \geq 0$ such that

$$
G(z)=(z-b)(\bar{b} z-1) p(z), \quad z \in \mathbb{D} .
$$

If $b=1$, we know that 1 is the Denjoy-Wolff point of the semigroup $\left(\eta_{t}\right)$ and (1) follows. Otherwise, $\lim _{r \rightarrow 1} p(r)=0$. Then the function $\varphi(z)=(1-p(z)) /(1+p(z))$ is a selfmap of the unit disc and $\lim _{r \rightarrow 1} \varphi(r)=1$. By [30. Proposition 4.13], the function $\varphi$ has angular derivative (possibly infinite) at 1 . Thus, $p$, and so $G$, has angular derivative at 1 . That is, the radial limit

$$
\lim _{r \rightarrow 1} \frac{G(r)}{r-1}
$$

exists. By (2), the limit is finite and again by [15, Theorem 1], we conclude that 1 is a boundary regular fixed point of the semigroup.

Theorem 4.7. Let $D \subset \subset \mathbb{C}^{n}$ be a strongly convex domain with smooth boundary, $F$ the infinitesimal generator of a semigroup $\left(\Phi_{t}\right)$ of holomorphic self-maps of $D$, and $p \in \partial D$. The following are equivalent:

(1) $p$ is a BRFP for $\left(\Phi_{t}\right)$.

(2) There exists $C>0$ such that for any Lempert projection device $\left(\varphi, \rho_{\varphi}, \widetilde{\rho}_{\varphi}\right)$ with $\varphi(1)=p$,

$$
\limsup _{(0,1) \ni r \rightarrow 1} \frac{\left|f_{\varphi}(r)\right|}{1-r} \leq C .
$$

Moreover, if $p$ is a BRFP for $\left(\Phi_{t}\right)$ with boundary dilatation coefficients $\alpha_{t}(p)=e^{\beta t}$ then the non-tangential limit

$$
A(\varphi, p):=\angle \lim _{\zeta \rightarrow 1} \frac{f_{\varphi}(\zeta)}{\zeta-1}
$$

exists, $A(\varphi, p) \in \mathbb{R}$ and $A(\varphi, p) \leq \beta$. Also, $\beta=\sup A(\varphi, p)$, with the supremum taken as $\varphi$ varies among all complex geodesics with $\varphi(1)=p$.

Proof. Suppose (1) holds. By Theorem 3.8 there exists $\beta \in \mathbb{R}$ such that $d\left(u_{D, p}\right)_{z}$. $F(z)+\beta u_{D, p}(z) \leq 0$ for all $z \in D$. Let $\left(\varphi, \rho_{\varphi}, \widetilde{\rho}_{\varphi}\right)$ be a Lempert projection device with $\varphi(1)=p$ and let $f_{\varphi}$ be the associated vector field. By Proposition 4.5, the map $f_{\varphi}$ is an infinitesimal generator and satisfies $d\left(u_{\mathbb{D}, 1}\right)_{\zeta} \cdot f_{\varphi}(\zeta)+\beta u_{\mathbb{D}, 1}(\zeta) \leq 0$ for all $\zeta \in \mathbb{D}$. By Theorem 3.8 , the semigroup generated by $f_{\varphi}$ in $\mathbb{D}$ has a BRFP at 1 with boundary dilatation coefficients $\alpha_{t}(1) \leq e^{t \beta}$. By Lemma 4.6 , the non-tangential limit $\angle \lim _{\zeta \rightarrow 1} f_{\varphi}(\zeta) /(\zeta-1)$ exists and it is a real number less than or equal to $\beta$, and thus (1) and part of the last statement are proved.

Suppose (2) holds. By Theorem 3.8, it is enough to show that there exists $\beta \in \mathbb{R}$ such that $d\left(u_{D, p}\right)_{z} \cdot F(z)+\beta u_{D, p}(z) \leq 0$ for all $z \in D$. Fix $z \in D$ and let $\varphi: \mathbb{D} \rightarrow D$ be the complex geodesic such that $\varphi(0)=z$ and $\varphi(1)=p$. By Proposition 4.5, the vector field $f_{\varphi}$ is an infinitesimal generator in $\mathbb{D}$. Hypothesis (2) and Lemma 4.6 imply that 1 is a BRFP for the semigroup generated by $f_{\varphi}$ with boundary dilatation coefficients less than or 
equal to $e^{C t}$. Therefore Theorem 3.8 applied to $f_{\varphi}$ yields $d\left(u_{\mathbb{D}, 1}\right)_{z} \cdot f_{\varphi}(\zeta)+C u_{\mathbb{D}, 1}(\zeta) \leq 0$ for all $\zeta \in \mathbb{D}$. By (4.7) it follows that $d\left(u_{D, p}\right)_{\varphi(\zeta)} \cdot F(\varphi(\zeta))+C u_{D, p}(\varphi(\zeta)) \leq 0$ for all $\zeta \in \mathbb{D}$, and thus in particular for $\zeta=0$ we have $d\left(u_{D, p}\right)_{z} \cdot F(z)+C u_{D, p}(z) \leq 0$ as needed.

Finally, notice that, again by Theorem 3.8 , the previous arguments also show that if $p$ is a BRFP with boundary dilatation coefficients $\alpha_{t}(p)=e^{\beta t}$ then $\beta$ is the supremum of all $A(\varphi, p)$.

Corollary 4.8. Let $F: D \rightarrow \mathbb{C}^{n}$ be the holomorphic infinitesimal generator of a semigroup $\left(\Phi_{t}\right)$ with a stationary point $p \in \partial \mathbb{B}^{n}$. Then for any Lempert projection device $\left(\varphi, \rho_{\varphi}, \widetilde{\rho}_{\varphi}\right)$ with $\varphi(1)=p$,

(1) $\angle \lim _{\zeta \rightarrow 1} f_{\varphi}(\zeta)=0$,

(2) $\angle \lim _{\zeta \rightarrow 1} f_{\varphi}(\zeta) /(\zeta-1)=A(\varphi, p)$ is a finite real number, $A(\varphi, p) \leq 0$ and the boundary dilatation coefficients $\alpha_{t}(p)=e^{t \beta}$ are such that $A(\varphi, p) \leq \beta \leq 0$ for all $\varphi$.

Moreover,

(a) if $F(z)=0$ for some $z \in D$ then there exists a complex geodesic $\varphi: \mathbb{D} \rightarrow D$ with $\varphi(1)=p$ such that $F(\varphi(\zeta))=0$ for all $\zeta \in \mathbb{D}$ and all points of $\varphi(\partial \mathbb{D})$ are stationary points for $\left(\Phi_{t}\right)$ with boundary dilatation coefficients $\alpha_{t}\left(\varphi\left(e^{i \theta}\right)\right)=1$ for all $\theta \in \mathbb{R}$; also $A\left(\varphi, \varphi\left(e^{i \theta}\right)\right)=1$ for all $\theta \in \mathbb{R}$,

(b) if $F(z) \neq 0$ for all $z \in D$ then $p$ is the Denjoy-Wolff point of $\left(\Phi_{t}\right)$.

Proof. Taking into account that if $z \in D$ then $F(z)=0$ if and only if $z \in \operatorname{Fix}\left(\Phi_{t}\right)$, the statement is a direct consequence of Theorem 4.7 and Proposition 2.9

\section{Boundary repelling fixed points and the non-linear resolvent}

In [31] Reich and Shoikhet proved the following result.

Theorem 5.1. Let $D \subset \mathbb{C}^{n}$ be a bounded convex domain (not necessarily strongly convex). Let $F: D \rightarrow \mathbb{C}^{n}$ be the holomorphic infinitesimal generator of a semigroup $\left(\Phi_{t}\right)$ of holomorphic self-maps of D. Then there exists a family $\left\{G_{t}\right\}$ of holomorphic self-maps of $D$, with $G_{0}=\mathrm{id}_{D}$, depending on the parameter $t \in[0,+\infty)$ such that for all $z \in D$ and $t \in[0,+\infty)$,

$$
G_{t}(z)-z=t F\left(G_{t}(z)\right)
$$

and for all $z \in D$,

$$
F(z)=\lim _{t \rightarrow 0^{+}} \frac{G_{t}(z)-z}{t} .
$$

Moreover, if $z, w \in D$ are such that $w-z=t F(w)$ then $w=G_{t}(z)$. 
The above family $\left\{G_{t}\right\}$ is called the non-linear resolvent of $F$. It reflects some dynamical properties of the semigroup. Indeed, by (5.1) (and uniqueness) it follows easily that

$$
\operatorname{Fix}\left(G_{t}\right)=\{z \in D: F(z)=0\}=\operatorname{Fix}\left(\Phi_{t}\right) .
$$

In [31, proof of Corollary 1.6], it is also proved that if $D \subset \subset \mathbb{C}^{n}$ is a strongly convex domain with smooth boundary and $\left(\Phi_{t}\right)$ has no fixed points in $D$, and $\tau \in \partial D$ is the Denjoy-Wolff point of the semigroup, then

$$
G_{t}\left(E_{D}(\tau, R)\right) \subseteq E_{D}(\tau, R)
$$

for all $R>0$ and all $t>0$. By Theorem $2.3 \tau$ is a stationary point for all $G_{t}$. Since $F$ has no zeros in $D$, we have $\operatorname{Fix}\left(G_{t}\right)=\emptyset$ and, by Proposition 2.9. we conclude that $\tau$ is the Denjoy-Wolff point of $G_{t}$ for all $t>0$. That is, the functions $\Phi_{t}$ and $G_{t}$ share the same Denjoy-Wolff point.

For boundary regular fixed points, we can prove:

Proposition 5.2. Let $D \subset \subset \mathbb{C}^{n}$ be a strongly convex domain with smooth boundary. Let $F: D \rightarrow \mathbb{C}^{n}$ be a holomorphic infinitesimal generator with associated semigroup $\left(\Phi_{t}\right)$, non-linear resolvent $\left\{G_{t}\right\}$, and $p \in \partial D$. Suppose there exists $\beta \in \mathbb{R}$ such that for any $t>0$ the point $p$ is a BRFP for $G_{t}$ with boundary dilatation coefficients $\alpha_{G_{t}}(p) \leq e^{t \beta}$. Then $p$ is a BRFP for $\left(\Phi_{t}\right)$ with boundary dilatation coefficients $\alpha_{t}(p) \leq e^{t \beta}$.

Proof. By Theorem 3.8 it is enough to prove that $d\left(u_{D, p}\right)_{z} \cdot F(z)+\beta u_{D, p}(z) \leq 0$ for all $z \in D$. Fix $z \in D$. Since $p$ is a BRFP for $\left\{G_{t}\right\}$ and $\alpha_{G_{t}}(p) \leq e^{t \beta}$, by Theorem 2.4, we have

$$
g(t):=u_{D, p}\left(G_{t}(z)\right)-e^{-t \beta} u_{D, p}(z) \leq 0
$$

for all $t \in[0, \infty)$. Since $g(0)=0$, using (5.1) and the fact that $G_{t}(z) \rightarrow z$ for $t \rightarrow 0^{+}$ by (5.2), we have

$$
\begin{aligned}
0 & \geq \lim _{t \rightarrow 0^{+}} \frac{g(t)}{t}=\lim _{t \rightarrow 0^{+}} \frac{u_{D, p}\left(G_{t}(z)\right)-u_{D, p}(z)}{t}+\beta u_{D, p}(z) \\
& =\lim _{t \rightarrow 0^{+}} \frac{u_{D, p}\left(t F\left(G_{t}(z)\right)+z\right)-u_{D, p}(z)}{t}+\beta u_{D, p}(z) \\
& =d\left(u_{D, p}\right)_{z} \cdot F(z)+\beta u_{D, p}(z),
\end{aligned}
$$

proving the statement.

Remark 5.3. If $p \in \partial D$ is a BRFP for $\left\{G_{t}\right\}$ with boundary dilatation coefficients $\alpha_{G_{t}}(p) \leq e^{\beta t}$ for some $\beta \in \mathbb{R}$, then (see Remark 2.5 $\mathrm{K}-\lim _{z \rightarrow p} G_{t}(z)=p$ for any $t \in[0,+\infty)$. In particular, from (5.1), it follows that for all $t>0$ and $R>1$,

$$
\lim _{G_{t}(K(p, R)) \ni z \rightarrow p} F(z)=0 .
$$

However, even in this case, $F$ might not have radial limit 0 at $p$. In fact, looking at the infinitesimal generator $\tilde{F}$ in Example 4.2, one easily sees that the non-linear resolvent $\left\{G_{t}\right\}$ has a BRFP at $e_{1}$ with boundary dilatation coefficients $\alpha_{G_{t}}\left(e_{1}\right)=1$ (because by construction $G_{t}(z)=z$ on a complex geodesic containing $e_{1}$ on its boundary). But $\tilde{F}$ does not have radial limit 0 at $e_{1}$. 
The converse to Proposition 5.2 is false, as the following example shows:

Example 5.4. Let $f(\zeta)=1-\zeta^{2}$. Then $f$ is the infinitesimal generator of a group of hyperbolic automorphisms in $\mathbb{D}$, with Denjoy-Wolff point 1 and boundary repelling fixed point -1 . It is easy to check that the non-linear resolvent of $f$ is given by

$$
G_{t}(z)=\frac{1}{2 t}\left(-1+\exp \left(\frac{1}{2} \log (1+4 t(t+z))\right)\right)
$$

for all $t>0$ and $z \in \mathbb{D}$. Now, a direct computation shows $G_{t}(-1)=\frac{|2 t-1|-1}{2 t} \neq-1$.

\section{Boundary behavior in the unit ball}

In this section we translate our results on BRFPs to semigroups on the unit ball $\mathbb{B}^{n} \subset \mathbb{C}^{n}$, where most expressions have computable forms.

In order to simplify our statements and without loss of generality, we will assume that, up to conjugation, the base point is $e_{1}=(1,0, \ldots, 0) \in \partial \mathbb{B}^{n}$.

Theorem 6.1. Let $F: \mathbb{B}^{n} \rightarrow \mathbb{C}^{n}$ be the infinitesimal generator of a semigroup $\left(\Phi_{t}\right)$ of holomorphic self-maps of $\mathbb{B}^{n}$. The following are equivalent:

(1) The point $e_{1} \in \partial \mathbb{B}^{n}$ is a BRFP for $\left(\Phi_{t}\right)$.

(2) There exists $C>0$ such that for all automorphisms $H=\left(H_{1}, \ldots, H_{n}\right): \mathbb{B}^{n} \rightarrow \mathbb{B}^{n}$ such that $H\left(e_{1}\right)=e_{1}$,

$$
\limsup _{(0,1) \ni r \rightarrow 1} \frac{\left|d\left(H_{1}\right)_{H^{-1}\left(r e_{1}\right)}\left(F\left(H^{-1}\left(r e_{1}\right)\right)\right)\right|}{1-r} \leq C .
$$

Moreover, if $e_{1}$ is a BRFP for $\left(\Phi_{t}\right)$ with boundary dilatation coefficients $\alpha_{t}\left(e_{1}\right)=e^{\beta t}$ then the non-tangential limit

$$
A\left(H, e_{1}\right):=\angle \lim _{\zeta \rightarrow 1} \frac{d\left(H_{1}\right)_{H^{-1}\left(\zeta e_{1}\right)}\left(F\left(H^{-1}\left(\zeta e_{1}\right)\right)\right)}{\zeta-1}
$$

exists, $A\left(H, e_{1}\right) \in \mathbb{R}$ and $A\left(H, e_{1}\right) \leq \beta$. Also, $\beta=\sup A\left(H, e_{1}\right)$, with the supremum taken as $H$ varies among all automorphisms of $\mathbb{B}^{n}$ with $H\left(e_{1}\right)=e_{1}$.

Proof. The result follows from Theorem 4.7 as soon as one realizes how Lempert projection devices in the unit ball are related to automorphisms of $\mathbb{B}^{n}$. Indeed, thanks to the double transitivity of the group of automorphisms of $\mathbb{B}^{n}$ on $\partial \mathbb{B}^{n}$, any complex geodesic $\varphi: \mathbb{D} \rightarrow \mathbb{B}^{n}$ of $\mathbb{B}^{n}$ passing through $e_{1}$ can be written as $\zeta \mapsto H^{-1}\left(\zeta e_{1}\right)$ for some suitable automorphism $H: \mathbb{B}^{n} \rightarrow \mathbb{B}^{n}$. The associated Lempert projection $\rho_{\varphi}$ is thus given by $\rho_{\varphi}(z)=H^{-1}\left(\left\langle H(z), e_{1}\right\rangle e_{1}\right)=H^{-1}\left(H_{1}(z), 0, \ldots, 0\right)$ and the left inverse is $\widetilde{\rho}_{\varphi}(z)=H_{1}(z)$. Therefore

$$
f_{\varphi}(\zeta)=d\left(\tilde{\rho}_{\varphi}\right)_{\varphi(\zeta)} \cdot F(\varphi(\zeta))=d\left(H_{1}\right)_{H^{-1}\left(\zeta e_{1}\right)} \cdot F\left(H^{-1}\left(\zeta e_{1}\right)\right),
$$


from which the statement follows.

In the statement of Theorem 6.1 the sufficient condition for $e_{1}$ to be a BRFP can be checked considering only the class of parabolic automorphisms $H$ (that is, those for which the boundary dilatation coefficient at $e_{1}$ is 1 ). For clarity, we examine in detail the case $n=2$. In that case we can limit ourselves to (parabolic) automorphisms of $\mathbb{B}^{2}$ of the form

$$
H_{s, \theta}(z)=\frac{\left(-s z_{2}+(1-\beta) z_{1}+\beta, e^{i \theta}\left(z_{2}+s z_{1}-s\right)\right)}{-s z_{2}-\beta z_{1}+1+\beta},
$$

where $\beta \geq 0, s=\sqrt{2 \beta}$ and $\theta \in \mathbb{R}$. Notice that $\left(H_{s, \theta}\right)^{-1}$ also has the same form of $H_{s, \theta}$ and the differential of $H_{S, \theta}$ at $e_{1}$ is

$$
d\left(H_{s, \theta}\right)_{e_{1}}=\left(\begin{array}{cc}
1 & 0 \\
s e^{i \theta} & e^{i \theta}
\end{array}\right) .
$$

If $\varphi: \mathbb{D} \rightarrow \mathbb{B}^{2}$ is a complex geodesic with $\varphi(1)=e_{1}$ and we write $\varphi^{\prime}(1)$ in projective coordinates as $\varphi^{\prime}(1)=\left[1: s e^{i \theta}\right]$ with $s \geq 0$ and $\theta \in \mathbb{R}$, the corresponding $H_{s, \theta}$ in 6.2 is such that $H_{s, \theta}(\mathbb{D} \times\{0\})=\varphi(\mathbb{D})$ and therefore, by uniqueness of complex geodesics, $\varphi(\zeta)=H_{s, \theta}(\psi(\zeta), 0)$ for some automorphism $\psi$ of $\mathbb{D}$. Thus, in the statement of Theorem 6.1 for $n=2$, it is enough to check condition 6.1) for $H$ belonging to the class of $H_{s, \theta}$ 's.

Theorem 6.1 and the previous observation can be used to obtain the boundary behavior of infinitesimal generators with some bounds on the image. To explain this, we prove the following corollary in $\mathbb{B}^{2}$, which can be easily generalized to $\mathbb{B}^{n}$ for any $n \geq 2$, and can be considered a Julia-Wolff-Carathéodory type theorem for infinitesimal generators.

Corollary 6.2. Let $F: \mathbb{B}^{2} \rightarrow \mathbb{C}^{2}$ be the infinitesimal generator of a semigroup with a BRFP at $e_{1}$. Suppose there exist a horosphere $E_{\mathbb{B}^{2}}\left(e_{1}, R\right)$ and two distinct points $a_{0}, a_{1} \in \mathbb{C}$ such that $F_{1}\left(E_{\mathbb{B}^{2}}\left(e_{1}, R\right)\right) \subset \mathbb{C} \backslash\left\{a_{0}, a_{1}\right\}$. Then

(1) $F_{1}$ has non-tangential limit 0 at $e_{1}$, that is, $\angle \lim _{z \rightarrow e_{1}} F_{1}(z)=0$,

(2) $\angle \lim _{\zeta \rightarrow 1}(1-\zeta) F_{2}\left(\frac{\left((1-\beta) \zeta+\beta, e^{i \theta}(s \zeta-s)\right)}{-\beta \zeta+1+\beta}\right)=0$ for all $\beta \geq 0, s=\sqrt{2 \beta}$ and $\theta \in \mathbb{R}$.

Proof. By the very definition of horospheres in $\mathbb{B}^{2}$, there exists a ball $B \subset E_{\mathbb{B}^{2}}\left(e_{1}, R\right)$ such that $B$ is tangent to $\mathbb{B}^{2}$ at $e_{1}$. Let $\left\{z_{k}\right\} \subset \mathbb{B}^{2}$ be any sequence converging to $e_{1}$ non-tangentially. Then the sequence $\left\{z_{k}\right\}$ is eventually contained in $B$. Hence

$$
k_{E_{\mathbb{B}^{2}}\left(e_{1}, R\right)}\left(z_{k},\left\langle z_{k}, e_{1}\right\rangle e_{1}\right) \leq k_{B}\left(z_{k},\left\langle z_{k}, e_{1}\right\rangle e_{1}\right) .
$$

For $k \rightarrow \infty$ we have $k_{B}\left(z_{k},\left\langle z_{k}, e_{1}\right\rangle e_{1}\right) \rightarrow 0$ because $z_{k} \rightarrow e_{1}$ non-tangentially in $B$ (and non-tangential sequences are special in the sense of Abate [1, Lemma 2.2.24]). Therefore

$$
\lim _{k \rightarrow \infty} k_{E_{\mathbb{B}^{2}}\left(e_{1}, R\right)}\left(z_{k},\left\langle z_{k}, e_{1}\right\rangle e_{1}\right)=0 .
$$

Now let $g:=\left.F_{1}\right|_{E_{\mathbb{B}^{2}}\left(e_{1}, R\right)}: E_{\mathbb{B}^{2}}\left(e_{1}, R\right) \rightarrow \mathcal{L}:=\mathbb{C} \backslash\left\{a_{0}, a_{1}\right\}$. By the monotonicity of Kobayashi distance we have

$$
\omega_{\mathcal{L}}\left(g\left(z_{k}\right), g\left(\left\langle z_{k}, e_{1}\right\rangle e_{1}\right)\right) \leq k_{E_{\mathbb{B}^{2}}\left(e_{1}, R\right)}\left(z_{k},\left\langle z_{k}, e_{1}\right\rangle e_{1}\right),
$$


and 6.4 forces

$$
\lim _{k \rightarrow \infty} \omega_{\mathcal{L}}\left(g\left(z_{k}\right), g\left(\left\langle z_{k}, e_{1}\right\rangle e_{1}\right)\right)=0 .
$$

Since $\mathcal{L}$ is hyperbolic, this means that if $g\left(\left\langle z_{k}, e_{1}\right\rangle e_{1}\right)$ tends to some $b \in \mathbb{C}$ then $g\left(z_{k}\right)$ must have the same limit as $k \rightarrow \infty$. By 6.1) it follows that $g\left(\zeta e_{1}\right)$ has non-tangential limit 0 at 1 . Since $\left\{z_{k}\right\}$ tends to $e_{1}$ non-tangentially, so does $\left\{\left\langle z_{k}, e_{1}\right\rangle e_{1}\right\}$. Then

$$
\lim _{k \rightarrow \infty} F_{1}\left(z_{k}\right)=\lim _{k \rightarrow \infty} g\left(z_{k}\right)=\lim _{k \rightarrow \infty} g\left(\left\langle z_{k}, e_{1}\right\rangle e_{1}\right)=0,
$$

proving that $F_{1}$ has non-tangential limit 0 at $e_{1}$.

As for (2), from Theorem 6.1 with $H=H_{s^{\prime}, \theta^{\prime}}\left(\right.$ for $s^{\prime} \geq 0, \theta^{\prime} \in \mathbb{R}$ ) as in (6.2), we have

$$
\angle \lim _{\zeta \rightarrow 1} d\left(\left(H_{s^{\prime}, \theta^{\prime}}\right)_{1}\right)_{H_{s^{\prime}, \theta^{\prime}}^{-1}\left(\zeta e_{1}\right)}\left(F\left(H_{s^{\prime}, \theta^{\prime}}^{-1}\left(\zeta e_{1}\right)\right)\right)=0 .
$$

By the very definition of $H_{s^{\prime}, \theta^{\prime}}$ (and keeping in mind that $\left(H_{s^{\prime}, \theta^{\prime}}\right)^{-1}=H_{s, \theta}$ for some $s \geq 0$ and $\theta \in \mathbb{R})$ an easy computation shows that

$d\left(\left(H_{s^{\prime}, \theta^{\prime}}\right)_{1}\right)_{H_{s^{\prime}, \theta^{\prime}}^{-1}\left(\zeta e_{1}\right)}\left(F\left(H_{s^{\prime}, \theta^{\prime}}^{-1}\left(\zeta e_{1}\right)\right)\right)=C(\zeta)\left[F_{1}\left(H_{s, \theta}\left(\zeta e_{1}\right)\right)+(1-\zeta) F_{2}\left(H_{s, \theta}\left(\zeta e_{1}\right)\right)\right]$,

where $C(\zeta)$ is a smooth function which tends to some real number $C \neq 0$ for $\zeta \rightarrow 1$. Thus, since $F_{1}$ has non-tangential limit 0 at $e_{1}$ by (1), statement (2) follows.

Example 6.3. The infinitesimal generator $F\left(z_{1}, z_{2}\right)=\left(0,-z_{2} /\left(1-z_{1}\right)\right)$ in Example 4.2 has the boundary behavior prescribed by Corollary 6.2 at $e_{1}$. Notice that $F_{2}$ does not have (non-tangential) limit 0 at $e_{1}$.

Acknowledgments. Part of this work was done in Seville where the first-named author spent the entire month of March 2006. He wants to sincerely thank the people at Departamento de Matemática Aplicada II at Escuela Superior de Ingenieros in Universidad de Sevilla for the gentle atmosphere and friendship he experienced there.

Research of the second-named and third-named authors was partially supported by the Ministerio de Ciencia y Tecnología and the European Union (FEDER) project BFM2003-07294-C02-02 and MTM2006-14449-C02-01, and by La Consejería de Educación y Ciencia de la Junta de Andalucía.

\section{References}

[1] Abate, M.: Iteration Theory of Holomorphic Maps on Taut Manifolds. Mediterranean Press, Rende, Cosenza (1989) Zbl 0747.32002 MR 1098711

[2] Abate, M.: The infinitesimal generators of semigroups of holomorphic maps. Ann. Mat. Pura Appl. 161, 167-180 (1992) Zbl 0758.32013 MR 1174816

[3] Aharonov, D., Elin, M., Reich, S., Shoikhet, D.: Parametric representations of semi-complete vector fields on the unit balls in $\mathbb{C}^{n}$ and in Hilbert space. Rend. Lincei Mat. Appl. (9) 10, 229-253 (1999) Zbl 1036.32017 MR 1767931

[4] Aharonov, D., Reich, S., Shoikhet, D.: Flow invariance conditions for holomorphic mappings in Banach spaces. Math. Proc. Roy. Irish Acad. 99A, 93-104 (1999) Zbl 0947.46033 MR 1883068 
[5] Aizenberg, L., Shoikhet, D.: Boundary behavior of semigroups of holomorphic mappings on the unit ball in $\mathbb{C}^{n}$. Complex Var. Theory Appl. 47, 109-121 (2002) Zbl 1030.32015 MR 1892512

[6] Berkson, E., Porta, H.: Semigroups of analytic functions and composition operators. Michigan Math. J. 25, 101-115 (1978) Zbl 0382.47017 MR 0480965

[7] Bisi, C., Bracci, F.: Linear fractional maps of the the unit ball: a geometric study. Adv. Math. 167, 265-287 (2002) Zbl 1008.32007 MR 1906258

[8] Bracci, F.: Commuting holomorphic maps in strongly convex domains. Ann. Scuola Norm. Sup. Pisa Cl. Sci. 27, 131-144 (1999) Zbl 0941.32018 MR 1658877

[9] Bracci, F.: Dilatation and order of contact for holomorphic self-maps of strongly convex domains. Proc. London Math. Soc. 86, 131-152 (2003) Zbl 1044.32015 MR 1971466

[10] Bracci, F., Trapani, S.: Notes on pluripotential theory. Rend. Mat. Appl. (7) 27, 197-264 (2007) Zbl 1145.32017 MR 2398425

[11] Bracci, F., Contreras, M., Díaz-Madrigal, S.: Classification of semigroups of linear fractional maps in the unit ball. Adv. Math. 208, 318-350 (2007) Zbl 1127.47038 MR 2304320

[12] Bracci, F., Contreras, M., Díaz-Madrigal, S.: Infinitesimal generators associated with semigroups of linear fractional maps. J. Anal. Math. 102, 119-142 (2007) Zbl 1144.32006 MR 2346555

[13] Bracci, F., Patrizio, G.: Monge-Ampère foliations with singularities at the boundary of strongly convex domains. Math. Ann. 332, 499-522 (2005) Zbl 1086.32028 MR 2181760

[14] Bracci, F., Patrizio, G., Trapani, S.: The pluricomplex Poisson kernel for strongly convex domains. Trans. Amer. Math. Soc. 361, 979-1005 (2009) Zbl 1165.32021 MR 2452831

[15] Contreras, M., Díaz-Madrigal, S., Pommerenke, Ch.: On boundary critical points for semigroups of analytic functions. Math. Scand. 98, 125-142 (2006) Zbl pre05239719 MR 2221548

[16] de Fabritiis, C.: On the linearization of a class of semigroups on the unit ball of $\mathbb{C}^{n}$. Ann. Mat. Pura Appl. 166, 363-379 (1994) Zbl 0820.32013 MR 1313813

[17] Demailly, J.-P.: Mesures de Monge-Ampère et mesures pluriharmoniques. Math. Z. 194, 519564 (1987) Zbl 0595.32006 MR 0881709

[18] Elin, M., Reich, S., Shoikhet, D.: Dynamics of inequalities in geometric function theory. J. Inequal. Appl. 6, 651-664 (2001) Zbl 1006.30009 MR 1888257

[19] Elin, M., Reich, S., Shoikhet, D.: Asymptotic behavior of semi-groups of $\rho$-nonexpansive and holomorphic mappings on the Hilbert ball. Ann. Math. Pura Appl. (4) 181, 501-526 (2002) Zbl pre02217039 MR 1939694

[20] Elin, M., Reich, S., Shoikhet, D.: A Julia-Carathéodory theorem for hyperbolically monotone mappings in the Hilbert ball. Israel J. Math. 164, 397-411 (2008) MR 2391157

[21] Elin, M., Shoikhet, D.: Dynamic extension of the Julia-Wolff-Carathéodory theorem. Dynam. Systems Appl. 10, 421-437 (2001) Zbl 0991.30502 MR 1858192

[22] Elin, M., Shoikhet, D.: Semigroups of holomorphic mappings with boundary fixed points and spirallike mappings. In: Geometric Function Theory in Several Complex Variables, World Sci., River Edge, NJ, 82-117 (2004) Zbl 1076.32007 MR 2115784

[23] Herzig, A.: Die Winkelderivierte und das Poisson-Stieltjes Integral. Math. Z. 46, 129-156 (1940) Zbl 0022.23802 MR 0001301

[24] Klimek, M.: Extremal plurisubharmonic functions and invariant pseudodistances. Bull. Soc. Math. France 113, 231-240 (1985) Zbl 0584.32037 MR 0820321

[25] Klimek, M.: Pluripotential Theory. London Math. Soc. Monogr. 6, Oxford Univ. Press (1991) Zbl 0742.31001 MR 1150978

[26] Kobayashi, S.: Hyperbolic Complex Spaces. Grundlehren Math. Wiss. 318, Springer (1998) Zbl 0917.32019 MR 1635983 
[27] Kopecká, E., Reich, S.: Hyperbolic monotonicity in the Hilbert ball. Fixed Point Theory Appl. 2006, Art. ID 78104, 15 pp. Zbl 1105.47046 MR 2210916

[28] Lempert, L.: La métrique de Kobayashi et la représentation des domaines sur la boule. Bull. Soc. Math. France 109, 427-474 (1981) Zbl 0492.32025 MR 0660145

[29] Lempert, L.: Intrinsic distances and holomorphic retracts. In: Complex Analysis and Applications 81 (Varna, 1981), Publ. House Bulgar. Acad. Sci., Sofia, 341-364 (1984) Zbl 0583.32060 MR 0883254

[30] Pommerenke, Ch.: Boundary Behaviour of Conformal Maps. Springer, Berlin (1992) Zbl 0762.30001 MR 1217706

[31] Reich, S., Shoikhet, D.: Semigroups and generators on convex domains with the hyperbolic metric. Rend. Lincei Mat. Appl. 8, 231-250 (1997) Zbl 0905.47056 MR 1631605

[32] Reich, S., Shoikhet, D.: Nonlinear Semigroups, Fixed Points, and Geometry of Domains in Banach Spaces. Imperial College Press, London (2005) Zbl 1089.46002 MR 2022955

[33] Rudin, W.: Function Theory in the Unit Ball of $\mathbb{C}^{n}$. Springer, Berlin (1980) Zbl 0495.32001 MR 0601594

[34] Shoikhet, D.: Semigroups in Geometrical Function Theory. Kluwer, Dordrecht (2001) Zbl 0980.30001 MR 1849612 